\title{
Zfp36l1 and Zfp36l2 balances proliferation and differentiation in the developing retina
}

\author{
Fuguo $\mathrm{Wu}^{1,2}$, Tadeusz Kaczynski ${ }^{1,3}$, Louise S. Matheson ${ }^{4}$, Tao Liư ${ }^{5}$, Jie Wang ${ }^{5}$, \\ Martin Turner ${ }^{4}$, Xiuqian Mu1,2,3* \\ ${ }^{1}$ Department of Ophthalmology/Ross Eye Institute, University at Buffalo, Buffalo, NY 14203, USA \\ ${ }^{2} \mathrm{New}$ York State Center of Excellence in Bioinformatics and Life Sciences, University at Buffalo, \\ Buffalo, NY 14203, USA \\ ${ }^{3}$ Neuroscience Graduate Program, University at Buffalo, Buffalo, NY 14203, USA \\ ${ }^{4}$ Laboratory of Lymphocyte Signaling and Development, The Babraham Institute, Babraham \\ Research Campus, Cambridge CB22 3AT, United Kingdom \\ ${ }^{5}$ Department of Biostatistics \& Bioinformatics, Roswell Park Comprehensive Cancer Center, \\ Buffalo, NY 14203, USA \\ * Corresponding author: Xiuqian Mu, 701 Ellicott Street, Buffalo, NY 14203, USA.
}

Email: xmu@buffalo.edu.

Author Contributions: Fuguo Wu performed experiments and analyzed data; Tadeusz Kaczynski performed experiments; Louise Matheson, Tao Liu, and Jie Wang performed bioinformatics analysis of data; Martin Turner contributed key mouse lines and analyzed data; Xiuqian Mu conceive the project, obtained funding, supervised the study, analyzed data, and wrote the paper.

Competing Interest Statement: The authors declare no competing interests.

Classification: Biological Sciences/Developmental Biology

Keywords: RNA-binding protein, mRNA stability, Retinal development, Cell differentiation, Cell proliferation 


\begin{abstract}
Both transcriptional and post-transcriptional regulation of gene expression play significant roles in diverse biological processes, but little is known about how posttranscriptional regulation impacts retinal development. Here we report our study of the function of two members of the TTP (tristetraprolin) mRNA binding protein family, Zfp36l1 and Zfp36I2, in the developing retina. TTP proteins are highly conserved $\mathrm{CCCH}$ zinc finger proteins, which carry out their functions by promoting target mRNA decay and modulating translation. We found that Zfp36l1 and Zfp36l2 were expressed in retinal progenitor cells (RPCs) during development and Müller glial cells and photoreceptors in the mature retina. Our analysis of the mutant retinas showed that, whereas the single knockout retinas were largely normal, the double knockout (DKO) retina showed decreased RPC proliferation and increased differentiation of multiple retinal cell types. RNA-seq analysis confirmed the imbalance of proliferation and differentiation in the DKO retina. Gene ontology and in silico target gene analysis indicates that Zfp36l1 and Zfp36l2 exert their function by directly regulating multiple classes of proteins, including components of multiple signaling pathways such as the sonic hedgehog pathway and the Notch pathway, cell cycle regulators, and most interestingly transcription factors directly involved in retinal differentiation. These results reveal a new tier of gene regulation controlling retinal development.
\end{abstract}




\section{Introduction}

The retina is an essential part of the visual system, serving as the receptor for light signals by transforming photons into electric signals and relaying them to the brain [1-3]. The function of the retina is carried out by the different retinal neurons, including cone and rod photoreceptors, horizontal cells, bipolar cells, amacrine cells, and retinal ganglion cells (RGCs), which form functional circuits through synapses and gap junctions in an exquisitely layered structure [1-3]. The only retinal glial cell type, Müller cells, is also essential for normal retinal function $[4,5]$. During development, all these cell types are generated from a single population of multipotent retinal progenitor cells (RPCs) [6, 7]. In the mouse, retinal cell differentiation takes place between embryonic day (E) 10 to about 10 days after birth (postnatal day 10, P10), with the different retinal cell types born in a conserved temporally sequential order, RGCs being the first type and Müller cells the last [8]. In the developing retina, RPCs either keep dividing or exit the cell cycle and differentiate into one of the seven retinal cell types. The balance between proliferation and differentiation is critical for retinal development to ensure proper numbers and proportions of the different retinal cell types. This balance is controlled through gene regulation during development, and many regulators of this process identified so far are transcription factors [9-18]. These intrinsic factors regulate the properties of RPCs and modulate their ability to proliferate and differentiate. Extrinsic factors also play important roles. The Notch pathway plays key roles in RPC proliferation and cell differentiation through lateral cell-cell interaction [12, 13, 18-25]. Differentiated cells, such as RGCs, regulate RPCs through a feedback mechanism by secreting signaling molecules including sonic hedgehog (Shh), Gdf11/Mstn (Gdf8), and Vegfa [26-31]. Nevertheless, the extrinsic factors eventually impose their action on RPCs by modulating intrinsic gene expression. Differentiation of the various retinal cell types are also subject to tight regulation, and many transcription factors functioning in the distinct retinal lineages have been identified. Some of these key transcription factors include Atoh7, Sox4/11/12, Pou4f2, and IsI1 for RGCs [32-36], Foxn4, Prox1, Ptf1a, and Tfap2a/b for amacrine cells and horizontal cells and amacrine cells [37-41], and Otx2, Crx, Neurod1, Nrl, and Nr2e3 for cone and rod photoreceptors [42-47].

Although gene regulation at the transcriptional level is essential for retinal development, post-transcriptional mechanisms likely are also critical. Posttranscriptional gene regulation has diverse biological functions and takes place at multiple points, including splicing, translocation, localization, decay, editing, and translation [48-50]. The focus of this current study is on two TTP (tristetraprolin ) mRNA-binding proteins, Zfp36l1 and Zfp36l2. TTP proteins are involved in multiple post-transcriptional processes including mRNA decay, translational control, and mRNA localization [51-56]. Among these processes, their roles in mRNA decay are best studied. TTP proteins bind to the adenylate- and uridylate (AU)-rich element (ARE) in the 3' UTR of a target mRNA via a conserved RNA binding domain composed of two tandem $\mathrm{CCCH}$ zinc fingers and recruit the Ccr4-NOT deadenylase complex, so that the adenosine nucleotides in the poly $(A)$ tail are 
processively removed and the mRNA is rapidly degraded [51, 56, 57]. Thus, the TTP proteins function by limiting the duration and amplitude of target genes, particularly in the feedback control of the responses to external signals during various biological processes such as inflammation [58]. In the mouse, there are four TTP members, Zfp36, Zfp36l1, Zfp36l2, and Zfp36l3 [56, 57, 59]. Since their RNA binding domains are highly conserved and all TTP members bind to the same ARE motif, significant redundancy among the TTP members exists. For example, Zfp36l1 and Zfp36l2 are redundantly involved in thymic development and T cell formation, B cell quiescence, and myogenesis [60-63].

Despite the broad roles post-transcriptional regulation mechanisms play in various biological processes, their functions in the neural system including the retina have not been well studied. However, the expression patterns of TTP proteins and functional studies suggest that they likely function critically in the neural system, including the retina [64-66]. In this study, we report that Zfp36/1 and Zfp36/2 are expressed in both the developing and mature retina in a highly specific fashion. By conditional gene targeting of the two genes in the embryonic retina, we show that Zfp36I1 and Zfp36I2 redundantly regulate the balance between retinal proliferation and differentiation. Using RNA-seq and in silico target analysis, we identify genes affected in the mutant retina and the likely targets among them, which indicate that Zfp36I1 and Zfp36l2 regulate retinal development via multiple classes of proteins, including components of multiple signaling pathways such as the sonic hedgehog pathway and the Notch pathway, cell cycle regulators, and most interestingly transcription factors directly involved in retinal differentiation. Our study thus reveals a novel layer of gene regulation controlling retinal development.

\section{Results}

\section{Expression of $Z f p 36 / 1$ and $Z f p 36 / 2$ in the developing retina}

In search of novel regulators of retinal development, we discovered significant levels of expression of Zfp36/1 and Zfp36/2 in our RNA-seq dataset obtained previously from the E14.5 retina [67]. Their expression was not affected by mutation of genes encoding some of the key regulators we have been studying, including Atoh7, Pou4f2, and Isl1 [67]. Single cell RNA-seq (scRNA-seq) analysis further demonstrated that both Zfp36/1 and Zfp36/2 were expressed in RPCs but not in differentiated neurons at E13.5 [67]. To confirm these findings and gain further information on their expression in the retina, we performed RNAscope in situ hybridization of Zfp36/1 and Zfp36/2 on wild-type retinal sections of different developmental stages. Both Zfp36/1 and Zfp36/2 displayed dynamic yet very similar retinal expression patterns at different developmental stages (Figure 1AJ). Consistent with findings from scRNA-seq analysis, both genes were expressed in RPCs, but not differentiated neurons, such as RGCs or photoreceptors, during development (E14.5 to P0) (Figure 1A-C, F-H). At early stages (e.g. E14.5), RNAseq data suggested that Zfp36/1 was expressed five times higher than Zfp36/2 [67], which was confirmed by in situ hybridization results (Figure 1A, F). At later stages (E17.5 and P0) the two genes were expressed at similar levels in RPCs 
(Figure 1B, C, G, H). In the mature retina, Zfp36/1 and Zfp36/2 exhibited cell typespecific expression patterns (Figure 1D, E, I, J). At P16, Zfp36/1 and Zfp36/2 were both expressed in photoreceptors and Müller cells as indicated by the locations of the signals (Figure 1D, I); whereas Zfp36/2 was expressed at much higher levels than Zfp36/1 in photoreceptors, they were expressed at similar levels in Müller cells (Figure 1D, I). At P90, the expression of Zfp36/2 remained at much higher levels than Zfp36/1 in photoreceptors; in contrast, in Müller cells, Zfp36/1 was expressed at much higher levels than Zfp36/2 (Figure. 1E, J). These results indicated that the two genes were expressed largely in the same cell populations but followed distinct dynamics throughout different stages.

\section{Retina-specific deletion of Zfp36/1 and Zfp36/2}

The expression patterns of Zfp36/1 and Zfp36/2 indicated that they might function in the developing and mature retina. To investigate that possibility, we deleted Zfp36/1 and Zfp36/2 in the retina by crossing the floxed Zfp36/1F and Zfp36/2F alleles [62] with retinal specific Cre lines Six3-Cre and Vsx2-Cre, since germline knockout mice of Zfp36/1 and Zfp36/2 are either embryonically or perinatally lethal [68-71]. Zfp36/1F/F, Zfp36/2F/F and Zfp36/1F/F;Zfp36/2F/F mice were phenotypically wild-type and served as controls throughout this study. Six3-Cre was frequently leaky [72], resulting in embryonic lethality and making it difficult to work with. Therefore, although we started with Six3-Cre and similar phenotypes were observed initially with the two lines, we performed most of the experiments with the Vsx2-Cre line [73]. As anticipated, both Six3-Cre and Vsx2-Cre-mediated deletion of Zfp36/1F/F and/or Zfp36/2F/F in the retina [73, 74]; Six3 deleted the floxed genes in the central region (data not shown), whereas Vsx2-Cre deleted in a mosaic fashion, as revealed by in situ hybridization (Figure 1K-M, N-P). Comparison with the wild-type retina suggested $\sim 50-80 \%$ of deletion efficiencies by Vsx2-Cre for both genes at E14.5 and E17.5 (Figure 1A, B, F, G, K, L, N, O). At P0, the proportions of mutant cells appeared to decrease when both genes were deleted together (Figure 1M, P), indicating a loss of mutant cells in the chimeric retina.

Histological analysis (H\&E staining) of mature retinal sections indicated that Zfp36/1F/F;Six3-Cre or Zfp36/2 F/F;Six3-Cre retinas were well laminated, and all of the retinal layers were comparable to those of their control (Zfp36/1F/F or Zfp36/2F/F) retinas at six months of age (Figure 1Q-T). Analysis of cell type-specific markers also did not reveal any overt defects. Zfp36/1F/F;Vsx2-Cre or Zfp36/2 F/F;Vsx2-Cre retinas also appeared normal. We also analyzed the embryonic (E14.5) Zfp36/1F/F; Six3-Cre retina and did not observe noticeable defects. The partial deletion by the two Cre lines was unlikely the reason for the lack of defects in the retina since they have been used extensively to delete other genes, leading to severe defects, and both Zfp36/1 and Zfp36/2 were deleted substantially (Figure 1A, B, F, G, K, L, N, O). Because the two genes had highly overlapping expression patterns in the 
retina, the lack of defects could have been due to their redundancy, as has been seen in other tissues [60-63].

\section{Zfp36I1 and Zfp36I2 are required for sufficient proliferation of RPCs}

To examine whether Zfp36l1 and Zfp36l2 function redundantly and to further investigate their roles in the retina, we generated conditional double knockout mice (DKO) with Vsx2-Cre. Since both genes were highly expressed in RPCs which are highly proliferative, we first sought to determine the effects of the deletion of Zfp36/1 and Zfp36/2 on RPC proliferation during development. For that purpose, we used BrdU incorporation to label S phase RPCs located in the neuroblast layer (NBL) in the Zfp36/1/2 DKO and control retinas. At E14.5, there was a significant decrease (17.5\% less) in the number of BrdU-positive cells in the Zfp361/2 DKO retina as compared to the control retina $(P<0.01)$ (Figure 2A, $\mathbf{F}, \mathbf{Q}$ ). Immunofluorescence staining for phosphorylated histone $3(\mathrm{pH} 3)$ also revealed a significant reduction (34.2\%) of M phase RPCs which are located at the apical side of the retina (Figure 2B, G, Q). At E17.5, more obvious decreases in the numbers of proliferating RPCs were observed; cells positive for three proliferation markers including BrdU, pH3, and PCNA all displayed marked reduction (Figure 2C-E, HJ). BrdU+ cells were decreased by $30.3 \%$ in the E17.5 DKO retina as compared to that of control $(\mathrm{P}<0.001), \mathrm{pH} 3$ positive cells were decreased by $54.2 \%$, and PCNA cells decreased by $37.5 \%$ (Figure 2Q). Consistent with the reduced numbers of RPCs, the NBL was thinner (Figure 2A, C, E, F, H, J). Noticeably, at E17.5, the thickness of the NBL became uneven and its edges were not as smooth as seen in the control retina (Figure $2 \mathbf{H}, \mathbf{J}$ ). The uneven thickness of the NBL in the DKO retina likely resulted from the mosaic deletion of Zfp36/1 and Zfp36/2 and thereby the differential proliferation rates of wild-type and mutant cells.

The thin NBL in the DKO retina also prompted us to examine whether there were changes in apoptosis in the DKO retina. We thus examined apoptotic cells in the DKO retina at E14.5, E17.5, and P0 by immunofluorescence staining of activated caspase 3 [29,74], which showed that there were indeed significant increases in the numbers of caspase-3+ cells in the Zfp36/1/2 DKO retina at all three stages, by 2.8, 4.0, and 4.1 fold respectively, as compared to the control retina (Figure $2 \mathrm{~K}$ $\mathbf{P}, \mathbf{R})$. These results indicated that Zfp36l1 and Zfp36l2 were required to maintain efficient proliferation and survival of RPCs during embryonic retinal development. Both reduced proliferation and increased apoptosis may have contributed to the reduced cell numbers in the NBL and the decreased proportion of mutant cells in the $\mathrm{PO}$ DKO retina (Figure $1 \mathrm{M}, \mathrm{P}$ ).

\section{Zfp36I1 and Zfp36I2 inhibit differentiation of both early and late retinal cell types}

Retinal cell differentiation occurs when selected RPCs exit the cell cycle and adopt one of the seven retinal cell fates. The seven different cell types are born in 
different time windows as two major waves of differentiation, with RGCs, horizontal cells, amacrine cells, and cones belonging to the first wave, and rods, bipolar cells, and Müller cells belonging to the second wave [8]. We thus examined how cell differentiation was affected in the DKO retina. For that purpose, we first examined the differentiation of RGCs, which belong to the first wave, using two markers Pou4f2 and IsI1 [28, 35, 75]. At E14.5, there was a significant increase $(45.5 \%)$ in the number of Pou4f2+ cells and thickness of the ganglion cell layer (GCL) in the Zfp36/1/2-deleted regions as indicated by anti-Zfp36l1/2 staining when compared to corresponding regions of the control retina (Figure $3 \mathbf{A}-\mathbf{F}, \mathbf{M}$ ). The number of $|\mathrm{s}| 1+$ cells was also noticeably increased by $21.1 \%$ (Figure $3 \mathbf{G}, \mathbf{J}$ ). We also examined Crx and Otx2, two markers for photoreceptors (mostly cones at this stage), but did not observe obvious differences in the numbers of Crx positive cells, but an 18.5\% decrease of Otx2 positive cells, between DKO and control retinas (Figure 3H, I, K, L, M). Next, we examined whether the differentiation of rods, which are born during the second wave, was affected at E17.5 by immunofluorescence staining of four photoreceptor markers, Crx, Otx2, Nr2e3, and Nrl. Whereas Crx and Otx2 are expressed in both rods and cones, Nr2e3, and $\mathrm{Nrl}$ are exclusive rod markers [42-46]. At E17.5, Crx exhibited a moderate increase (18.7\%) (Figure 4A, B, Q), whereas Otx2 showed a slight but statistically insignificant increase, in the DKO retina (Figure $\mathbf{4 C}, \mathbf{D}, \mathbf{Q}$ ). However, cells expressing $\mathrm{Nr} 2 \mathrm{e} 3$ and $\mathrm{Nrl}$ increased markedly, by $82.0 \%$ and $73.1 \%$ respectively, indicating that rod differentiation increased in the DKO retina (Figure 4E-Q). Similar to what was observed with RGC markers, the increased rods occurred more apparently in the mutant regions as indicated by co-staining for Zfp36l1/2 (Figure 4H-J, N-P). These results indicated that the inactivation of Zfp36/1/2 led to increased differentiation of at least two cell types, RGCs and rods, at two developmental stages. Thus, Zfp36l1 and Zfp36l2 normally inhibit the differentiation of these two cell types.

\section{RNA-seq reveals a global impact of Zfp36/1 and Zfp36I2 on retinal cell proliferation and differentiation}

Although marker analysis revealed that there was decreased proliferation and increased differentiation in the Zfp36/1/2 DKO retina, it did not provide a picture of global changes in gene expression caused by the absence of these two proteins, as only a small number of markers could be analyzed with limited sensitivity. To gain further insights into the genes/pathways regulated by Zfp36I1 and Zfp36I2 in retinal development, we performed RNA-seq to compare the transcriptomes of wild-type and DKO retinas from different stages including E14.5, E17.5, and P0. Using a minimum fold change of 1.25 and a maximum adjusted p value of 0.05 , we identified 625 upregulated and 466 downregulated genes at E14.5, 450 upregulated genes and 378 downregulated genes at E17.5, and 448 upregulated gene and 497 downregulated genes at P0 (Figure 5A, Suppl. Tables 1-3). A relatively lower fold change cutoff (1.25) was used in consideration of two factors: posttranscriptional regulation tends to affect expression to lesser degrees than 
transcriptional regulation [76, 77], and Vsx2-Cre deleted the two floxed genes partially in a mosaic fashion, which likely further dampened the detected fold changes.

The RNA-seq analysis not only confirmed changes of the RGC and photoreceptor maker genes examined in the immunofluorescence analysis but also revealed many more marker genes for other cell types that exhibited upregulation in the DKO retina (Figure 5A, B, Suppl. Tables 1-3). By comparing the upregulated genes in all three stages (E14.5, E17.5, and P0) with lists of genes enriched in RGCs, horizontal and amacrine cells, and photoreceptors obtained by single cell RNA-seq at E13.5 [67], we identified large numbers of upregulated genes in the DKO retina that were enriched in the different cell types. For example, at E14.5, 176 upregulated genes were enriched in at least one of these cell types. Among them, 77 were enriched in RGCs only, 20 were enriched in horizontal and amacrine cells, 23 were enriched in photoreceptors, and 56 were enriched in more than one cell type (Suppl. Table 4). At E17.5 and P0, many upregulated genes enriched in all three cell types were also identified (Suppl. Table 4). These genes likely did not cover all the cell type specific genes that were differentially expressed, particularly those expressed in horizontal, amacrine cells and photoreceptors at later stages, since the enriched gene list was generated from the E14.5 retina. Nevertheless, these results indicated that Zfp36l1 and Zfp36I2 influenced the differentiation of all the four cell types generated in the first wave and rods, which are generated during the early phase of the second wave. Some of the cell type specific upregulated genes included Pou4f1, Gal, Is/1, and Pou4f2 for RGCs, Ptf1a, Tfap2b, Prox1, and Vtn for horizontal and amacrine cells, and Opn1sw, Nr2e3, and Nrl for cone and rod photoreceptors (Figure 5A, B, Suppl. Tables 14) [67]. Consistent with the immunofluorescence marker analysis, RNA-seq also revealed that upregulation of these cell type specific genes was most pronounced in the corresponding time windows when the relevant cell types were normally generated, although many genes had changed at more than one time points (Suppl. Table 1-4). For example, upregulation of RGC marker genes, including Pou4f1, Pou4f2, Gal, and Is/1, and genes for horizontal and amacrine cells such as Ptf1a, Tfap2b, Prox1, and Vtn was observed mostly at E14.5, whereas photoreceptor marker genes showed most changes at E17.5 (Figure 5B). On the other hand, the changes of the marker genes for individual cell types were not universal; only subsets of marker genes for each subtype displayed significant changes, and the degree of changes as manifested by fold changes varied considerably. For example, and consistent with the immunofluorescence results, Crx and Otx2 showed only moderate or no significant increases in the DKO retina, whereas Opn1sw, Nr2e3, and $\mathrm{Nrl}$ all displayed marked increases (Figure 5B, Suppl. Tables 1-3). This was also true to RGC marker genes: despite the increased expression of many RGC marker genes, particularly at E14.5, many other genes, including many widely used ones such as Gap43, Stmn2, Ebf1, Ebf3, Pou6f2, Rbpms, and Sncg, did not change in the DKO retina (Suppl. Tables 1-4). These observations indicated that Zfp36l1 and Zfp36l2 regulated only certain aspects of retinal cell differentiation through modulating subsets of cell type 
specific genes. Since Zfp36l1 and Zfp36l2 promote mRNA degradation and their absence leads to increased stability of target mRNAs, many of these upregulated marker genes in the DKO retina, particularly those expressed early during differentiation, could be their direct targets.

To further decipher how Zfp36l1 and Zfp36I2 balance retinal cell proliferation and differentiation, we performed gene ontology (GO) analysis by DAVID on the differentially expressed genes [78]. Since there are significant overlaps between the gene lists from different stages (Suppl. Figure 1), we combined these lists from E14.5, E17.5, and P0 to generate a non-redundant DEG list and then performed GO term enrichment analysis on the up- and downregulated genes separately. Consistent with the increased cell differentiation and upregulation of many marker genes, the top enriched GO terms of the upregulated genes included visual perception, axonogenesis, photoreceptor cell maintenance, axon guidance, cell adhesion, retinal development, and other terms of general neural development (Table 1). On the other hand, GO terms enriched with the downregulated genes included two major categories: those related to protein translation and those related to DNA repair and replication; 75 genes were associated with the $\mathrm{GO}$ term Translation, 33 with DNA Replication, and 63 with Cell Cycle (Table 1). Some of the genes associated with DNA replication and cell cycle included those encoding a DNA ligase (Lig1), DNA polymerases (e.g. Pola1, Pola2), members of the MCM complex (e.g. Mcm2-7, 9, 10), Cdc25b, and cyclin D1 (Ccnd1), and almost all of them were downregulated in all the three stages (Figure 5C, Suppl. Tables 1-3). These results indicated Zfp36I1 and Zfp36I2 played central roles in regulating the two biological processes critical for active proliferation in the developing retina. These downregulated genes likely were the underlying causes of the reduced proliferation in the DKO retina. However, these genes were unlikely directly regulated by Zfp36l1 and Zfp36l2, since the two proteins promote the degradation of target mRNAs, and thus their direct targets should be upregulated in the DKO retina.

\section{Zfp36I1 and Zfp36I2 modulate multiple signaling pathways in the developing retina}

Interestingly, genes encoding components of the Shh and Notch pathways, two major pathways promoting RPC proliferation, were altered (Figure 6 A, B, Suppl. Table 1-3). The Shh pathway regulates the balance between proliferation and differentiation via a feedback mechanism; Shh molecules secreted from differentiated cells (RGCs) act on RPCs via their receptor Smoothened (Smo) to promote RPC proliferation and inhibit the production of RGCs [29-31]. The changes in the Shh pathway mostly occurred at E14.5; most of the genes show less degree of changes at E17.5 or P0 (Figure 6A, Suppl. Tables 1-3). In the E14.5 Zfp36/1/2 DKO retina, Smo and Gli1, two target genes of the pathway, were significantly downregulated, but there was no significant change in Shh expression (Figure 6A). Nevertheless, Hhip, which encodes an Shh antagonist [79], was 
significantly upregulated (Figure 5A, Figure 6A, Suppl. Table 1). By in situ hybridization, we confirmed these changes in the Zfp36/1/2 DKO retina (Figure 6C). Further, we found that Hhip was expressed in RPCs in which the Shh signaling took place and Zfp36l1 and Zfp36l2 were expressed (Figure 6C), indicating that Hhip likely served as a mediator in the regulation of the Shh pathway by the two mRNA binding proteins. Consistent with this idea, using RegRNA2.0 [80] (regrna2.mbc.nctu.edu.tw/), we identified four regions that contained ARE motifs in the 3' UTR of the Hhip mRNA (Figure 6D). Noticeably, the ARE motifs were highly conserved across nine vertebrate species including chicken and multiple mammals (Figure 6D). For example, the three motifs in first region were all highly conserved and two of them were essential invariant in all these species (Figure 6D). Thus, Zfp36l1 and Zfp36l2 likely regulated the Shh pathway by directly modulating the mRNA levels of Hhip. In the DKO retina, the absence of Zfp36I1 and Zfp36I2 resulted in increased Hhip, which inhibited the function of Shh and thus reduced the strength of this pathway and RPC proliferation.

The Notch pathway plays pleiotropic roles in the retina through the multiple Notch ligands, receptors, and downstream effectors expressed in the developing retina $[12,13,18,20,22,81-84]$. One critical role this pathway plays is to balance proliferation and differentiation; it is essential for RPC proliferation and is turned off before differentiation [12, 18, 19, 23, 84, 85]. Multiple genes of the Notch pathway had altered expression levels in the Zfp36/1/2 DKO retina (Figure 6B). Consistent with the previous report that its mRNA is a target of Zfp36I1 and Zfp36I2 [62], Notch1 was upregulated, but only moderately (1.23 fold, adjusted $p$ value = 0.013). Two Notch ligand genes, DII3, and Jag1, were also upregulated. Interestingly, Hes1 and Hes5, two target genes of the Notch pathway, were differentially affected in the DKO retina; Hes5 was upregulated whereas Hes1 was downregulated. An additional target gene of the Notch pathway, Hey2, was also upregulated although it was expressed at much lower levels than Hes1 and Hes5 (Figure 6B, Suppl. Tables 1-3). Changes of the Notch pathway genes in the DKO retina also occurred mostly at E14.5, although the downregulation of Hes1 and Hey2 persisted at later stages (Figure 6B). These results indicated that Zfp36I1 and Zfp36l2 modulated the Notch pathway in a complex fashion by differentially influencing the different component genes. Nevertheless, downregulation of Hes1 may have contributed to the reduced RPC proliferation in the Zfp36/1/2 DKO retina. This downregulation may have resulted from the downregulation of the Shh pathway, as the two pathways interact and Hes1, but not Hes5, is also dependent on Shh [31, 86, 87].

Noticeably, many genes encoding other secreted molecules and even their receptors, including Dkk3, Fgf15, Fgf9, Fgfr1, Gdf11 lgf2, Igfbp5, Myostatin (Mstn), and Vegfa, were also upregulated in the DKO retina (Figure 6E). Among these molecules, Gdf11 and Vegfa have been shown to promote proliferation and inhibit differentiation [26, 27], and Vegfa mRNA is a known target of the TTP proteins [68, $88,89]$. The mRNAs of many of the other secreted molecules could also be directly regulated by Zfp36I1 and Zfp36I2 in the developing retina. 


\section{In silico identification of direct target mRNAs of Zfp36I1 and Zfp36I2 in the retina}

To gain further insights into the mechanisms by which Zfp36l1 and Zfp36I2 exert their functions, we attempted to identify the targets of these two proteins. Target genes of Zfp36l1 in the thymus have been experimentally identified by iCLIP [63]. Although the thymus is a very different tissue from the retina, we reasoned that there might still be some target genes expressed in both tissues, and thus compared the iCLIP list with the six DEG lists, including the up- and downregulated DEGs from all the three stages. We were able to find genes on all six lists that were present on the iCLIP list, with the most found in the E14.5 upregulated list (Suppl. Tables 1-3, Figure 7A). Since Zfp36l1 and Zfp36l2 regulate mRNA degradation, real targets in the mutant retina should be enriched in the upregulated gene lists as compared to what would be found by chance. To test whether this was the case, we generated 100 control gene sets for each DEG list that matched the number of genes, their expression levels in thymus, and their 3 UTR lengths, examined the presence of target genes from the thymus in them and determined the median as well as the 5th and 95th percentile gene numbers (Figure 7A). The number of thymus target genes in the E14.5 upregulated DEG list was almost twice as that of the 95th percentile of the corresponding control gene sets, whereas the number in the E14.5 downregulated gene list was much smaller than the 5th percentile number of the controls, indicating real targets were indeed enriched in the upregulated DEGs, but depleted in the downregulated DEGs. The same trend of enrichment was observed in both the E17.5 and P0 upregulated DEG lists but to much lesser degrees. Depletion was observed in E17.5, but not P0, downregulated gene list. (Figure 7A).

To expand our view on the Zfp36|1/Zfp36l2 target genes in the retina, we next examined the presence of AREs in the 3' UTRs of the DEGs, assuming their presence was a strong indicator for real targets. Although TATTTATT is the initially identified core ARE motif for the TTP proteins, closely spaced TATTs can also be efficiently bound by them $[90,91]$. Thus we search for both TATTTATT motifs and adjacent TATT motifs separated by less than 4 bases. As expected, we found more genes containing adjacent TATT motifs than those with TATTTATT motif in each of the DEG lists, since the former gene sets included the latter ones (Figure 7B, C). We then did enrichment analysis for the ARE containing genes with the same 100 control gene sets mentioned above for each of the DEG gene lists. Similar to what was observed with the thymus target genes, ARE-containing genes, either containing just TATTTATT motifs or adjacent TATT motifs spaced by less than 4 bases, were highly enriched in the E14.5 upregulated DEG list and depleted in the E14.5 downregulated DEG list (Figure 7B, C). Again, enrichment was also observed in the upregulated DEG lists for E17.5 and P0, but no enrichment or depletion in the downregulated genes at the two stages. The relatively weak enrichment of target genes at the two later stages does not necessarily suggest that Zfp36l1 and Zfp36l2 play lesser roles; rather; it may have reflected the 
secondary effects to the retina, such as cell death, caused by the deletion of the two genes at early stages.

The enrichment of the thymus target genes and those containing AREs in the upregulated DEG lists indicated these genes were highly likely real targets of Zfp36l1 and Zfp36l2, and a combined total of 602 presumed target genes were thus identified from the three stages (Suppl. Tables 5). The function of these candidate target genes could shed clues on how Zfp36I1 and Zfp36I2 exert their functions in the developing retina. Consistent with previous reports that TTP proteins often regulate signaling molecules, many of the secreted molecules including those discussed above, but not all, were indeed on the presumptive list of retinal target genes. Such target genes Dkk3, Gdf11, Igfbp5, Tgfb2, Cxcl12, Mstn, Pdgfa, Ptn, Fgf12, Pgf, Hbegf, Vegfa, and Manf, but not Fgf15, Fgf9, Kitl, Igf2, Inhbb, and Fgf4, although the significance for many of these molecules in retinal development has not been well studied. Several genes encoding key negative regulators of cell cycle progression, including Cdkn1a, Cdkn1c, Cdkn2d, and Cdk2ap2, were on the presumed target list, indicating Zfp36I1 and Zfp36I2 also modulate cell cycle progress and thereby proliferation directly. Also on the list were genes involved in axon genesis and pathfinding, including Sema6c, Epha4, Sema6d, Sema3g, Cxcr4, Sema3e, Sema4g, L1cam, Epha3, and Epha2, but genes participating in other aspects of differentiation were not particularly enriched. For example, most upregulated genes involved in RGC and photoreceptor differentiation and function did not seem to be direct targets. Remarkably, a large set (64) of transcription factor genes involved in differentiation of the various retinal cell types, many of which discussed above (Figure 5B, Suppl. Table 6), were on the target list of Zfp36l1 and Zfp36l2. Examples of these genes included Is/1, Pou4f2, Pou4f1, Pou4f3, Irx2 and Sox11 for RGCs, Ptf1a, Prox1, and Tfap2b for horizontal cells, Crx, Neurod1, Neurod4, and Nr2e3 for photoreceptors. Moreover, the majority of AREs (43 out 64) present in the 3' UTRs of these transcription factor genes were highly conserved among vertebrate species from chicken to mammals; this was exemplified by the complete conservation of ARE motifs in five such transcription factor genes (Suppl. Table 6, Figure 7D). This was likely an underestimate since we did not examine variant ARE motifs that can still be bound by the TTP proteins. Thus, one major plausible mechanism for Zfp36l1 and Zfp36I2 to modulate retinal differentiation is by directly promoting the decay of the mRNAs of a myriad of cell type-specific transcription factors.

\section{Postnatal Zfp36/1/2 DKO retina displays dysplasia and degeneration}

To investigate how the developmental defects affected the eventual outcome of the DKO retina, we examined its morphological changes at different postnatal stages. At P0, the NBL continued to be thinner as compared to the wild-type and was uneven in thickness with jagged edges (Figure 8A, $\mathbf{A}^{\prime}$ ). At P5, the normal structure of the mutant retina was disrupted more pronouncedly and rosettes were 
observed in the outer part of the retina (Figure 8B, B'). At P22, when the laminar structure had formed normally in the control retina, the DKO retina displayed more severe dysplasia with disruptions in both the outer nuclear layer (ONL) and inner nuclear (INL); rosette structures persisted in the ONL, and the INL intruded into the $\mathrm{ONL}$ and the two layers fused in some areas (Figure $\mathbf{8 C}, \mathbf{C}^{\prime}$ ). The inner plexiform layer (IPL) and GCL appeared largely undisrupted. At P90, the DKO retina became severely degraded, as manifested by the thinning and even disappearance of the ONL. Disruptions of the inner part of the retina such as the IPL and GCL were also observed (Figure 8D, D').

We then performed immunofluorescence labeling using cell type-specific markers to examine how the different retinal cell types formed at P16 (Figure 8E-L, E'-L'). These markers included rhodopsin (rods), cone arrestin (CAR, cones), calbindin (horizontal cells and amacrine cells), Vsx2 (bipolar cells), Pax6 and (amacrine cells and RGCs), Syntaxin (amacrine cells), Vimentin (Müller cells), and Pou4f1 (RGCs). We observed that all these cell types formed in Zfp36/1/2 DKO retinas (Figure 8E-L, E'-L') and had similar numbers as compared to the wild-type retina (Figure 8M). However, many cell types including rods, cones, horizontal cells, bipolar cells, and RGCs were displaced and were even found across the whole thickness of the retina (Figure 8E'-L').

Since the cellular dysplasia in the DKO retina occurred in P0 and P5 retinas already, before Zfp36l1 and Zfp36l2 were robustly expressed in photoreceptors and Müller cells, the postnatal defects could be caused by the developmental defects at the earlier stages. Since mutant cells in the DKO retina proliferated slower and many of them died (Figure 2), we examined how the mutant cells persisted in the postnatal retina by in situ hybridization. As shown earlier the proportions of mutant cells were much reduced already at P0 (Figure 1M, P). At P5, both Zfp36/1 and Zfp36/2 continued to be expressed in RPCs, which had reduced to a narrow band, in the wild-type retina (Figure $\mathbf{8 N}, \mathbf{0}$ ). In the DKO retina, unlike at earlier stages when wild-type and mutant cells form mosaics (Figure 1KP), only occasional gaps of mutant cells not expressing Zfp36l1 or Zfp36l2 were observed (Figure 8N' $\mathbf{0}$ '), suggesting that that the DKO retina was composed mostly of wild-type cells at this stage and the mutant cells were largely lost. These findings indicated that the postnatal dysplasia and degeneration were likely caused by the early developmental defects, particularly the death of the mutant cells, and did not reflect the direct functions of Zfp36l1 and Zfp36I2 in photoreceptors and Müller cells in the mature retina.

\section{Discussion}

In this paper, we demonstrate that Zfp36/1 and Zfp36/2, which encode two TTPfamily mRNA binding proteins, are expressed in RPCs during development, and in photoreceptors and Müller cells in the mature retina, with distinct but overlapping temporal and spatial patterns. Whereas the single knockout retinas of these two genes are largely normal, double knockout results in severe defects during 
development, indicating that Zfp36l1 and Zfp36l2 function redundantly. The redundancy among the TTP proteins, including between Zfp36I1 and Zfp36I2, have been reported in other systems and thus appears to be a common paradigm for this family of mRNA binding proteins.

Our results demonstrate that the major function of Zfp36l1 and Zfp36I2 in the developing retina is to balance proliferation and differentiation, promoting proliferation whereas inhibiting differentiation. The two proteins are required for sufficient proliferation and survival of RPCs. The inhibition of differentiation is not cell type-specific, since RNA-seq reveals that all retinal cell types generated at the developing stages studied here increase in the DKO retina. A critical question is how Zfp36l1 and Zfp36l2 carry out their functions. Our analysis of the potential target genes of Zfp36l1 and Zfp36l2 suggests that the two proteins exert their functions by regulating genes encoding multiple classes of proteins (Figure 9).

In line with what has been known on the functions of the TTP in regulating various signaling pathways, Zfp36l1 and Zfp36l2 modulate several signaling pathways in the developing retina. Both the Shh pathway and the Notch pathway play essential roles in balancing proliferation and differentiation. Since they both are affected in the DKO retina, Zfp36l1 and Zfp36l2 likely exert their functions, at least in part, by modulating these two pathways. Shh, which is produced by RGCs, promotes progenitor proliferation through a feedback mechanism. The gene regulatory cascade promoting RGC differentiation, which is composed of such key regulator as Atoh7, Sox11, Pou4f2, and Isl1, also activate Shh expression, and Shh then acts on RPCs by binding to its receptor Smoothened (So), which relieves the inhibition of the pathway by Patched 1 and Patched 2, and activates the effector genes such Gli1 and Ccnd1 to promote RPC proliferation and inhibit RGC differentiation [28-31, 35, 67, 92, 93] (Figure 9). Interestingly, Shh also promotes the expression of Hhip, which is an antagonist of Shh [67] (Figure 9). Our finding that Zfp36l1 and Zfp36I2 likely promote the degradation of Hhip mRNA adds another aspect to the pathway; a double inhibitory mechanism seems to be at work to regulate this signaling pathway, and thereby the balance of proliferation and differentiation (Figure 9). The Notch pathway is also required for efficient RPC proliferation. As in T cells, Notch1 mRNA likely is a direct target of Zfp36I1 and Zfp36l2. However, the Notch pathway seems to be modulated by these two proteins in a complex fashion, as demonstrated by the opposite responses of Hes1 and Hes5, two downstream targets and effectors of the pathway. The downregulation of Hes1 may be due to the interaction with the Shh pathway, as Hes1 is also dependent on Shh signaling in the retina [31, 86, 87], and thus the net effects in the DKO retina, namely the decreased proliferation and increased differentiation, are resulted from not just changes to these two pathways, but also their interactions (Figure 9). As indicated by the many other potential target genes encoding signaling molecules, additional pathways, including the Vegfa pathway and Gdf11/Mstn pathway, are also directly modulated by Zfp36l1 and Zfp36l2. As confirmed or likely targets, both Vegfa and Gdf11 inhibits differentiation and promote proliferation and interact with the Notch pathway [26, 27]. The 
upregulation of these two molecules in the DKO retina likely also contributes to the final phenotypical outcome.

Zfp36l1 and Zfp36l2 seem also to regulate proliferation and differentiation directly. Geens encoding four key negative cell cycle regulators, Cdkn1a (p21), Cdkn1c (p57), Cdkn2d (p19), and Cdk2ap2 (p14), are likely direct targets of Zfp36l1 and Zfp36l2. Thus, Zfp36l1 and Zfp36l2 directly promote proliferation by reducing the levels of these cyclin dependent kinase inhibitors (Figure 9). Indeed, some of them have been shown to be critical for retina progenitor cells to exit the cell cycle for differentiation [94]. Nevertheless, the most interesting yet somewhat unexpected finding from this study is that Zfp36l1 and Zfp36I2 repress retinal differentiation by directly regulating a large number of transcription factors required for different lineages (Figure 9). This conclusion is strongly supported by not just their upregulation in the DKO retina, the presence of AREs in their mRNA 3' UTRs, but also the deep conservation of these AREs from chicken to mammals. This novel finding expands our understanding of the mechanisms by which TTP proteins exert their biological functions and neural differentiation is regulated. Overall our results uncover a novel tier in the complex gene regulatory network in retinal development.

Zfp36l1 and Zfp36l2 regulate retinal development in a tissue-specific fashion. Many genes, e.g. Bcl2 and Cdk6, are regulated in different directions from other tissues [95, 96] (Suppl. Table 1-3). Further, many of the downregulated genes involved in cell division in the DKO retina are upregulated in the $B$ cell lineage when the two genes are knocked out [61]. Additionally, Zfp36l1 and Zfp36I2 have been reported to inhibit cell cycle progression [97-99], instead of promoting it as we demonstrate in the retina. These findings are consistent with the idea that the TTP proteins regulate target mRNAs in a tissue/cell type specific manner, and even the same mRNA can be differentially regulated in different cell types [100], although the underlying mechanisms are unknown. The tissue-specific expression of the TTP genes and their targets likely play key roles in the specificities, but other mechanisms such as protein phosphorylation and protein-protein interactions may also be involved [52, 101-103]. It's worth noting that although the current study is largely based on the function of Zfp36l1 and Zfp36I2 in promoting mRNA decay, the two proteins may also function via other mechanisms such as translational control and mRNA localization [52, 53].

Roles of Zfp36l1 and Zfp36l2 in the late born-cell types, such as bipolar cells and Müller cells, were not addressed in this study due to the early expression of the Cre lines used. By the time these two cell types are normally generated (P3-P10) [8], most mutant RPCs are lost in the DKO retina. This issue may be addressed in the future by using inducible RPC-specific Cre lines, which will allow for specific deletion of these two genes in RPCs in the relevant time windows. Zfp36/1 and Zfp36/2 continue to be expressed in photoreceptors and Müller cells of the mature retina. The postnatal defects of the DKO retina, namely cellular dysplasia and degeneration, are likely consequent from earlier developmental problems, particularly loss of the mutant retinal cells due to impaired proliferation and increased apoptosis. Nevertheless, given the highly specific expression of these 
two genes in the mature retina, they likely play specific roles in photoreceptors and Müller cells as well. These potential roles need to be investigated by conditional inactivation of these two genes in photoreceptors and Müller cells in the postnatal retina.

\section{Materials and Methods}

\section{Mice}

The floxed Zfp36/1 allele $\left(Z f p 36 / 1^{F}\right)$ and Zfp36I2 allele $\left(Z f p 36 / 2^{F}\right)$ were reported previously [62]. The Six3-Cre transgenic line has also been described before [104]. The Vsx2-Cre (Chx10-Cre) line was obtained from the Jaxson Laboratory [73]. All mice were maintained in a C57/BL6 $\times 129$ genetic background. All procedures using mice conformed to the US Public Health Service Policy on Humane Care and Use of Laboratory Animals and were approved by the Institutional Animal Care and Use Committees of the Roswell Comprehensive Cancer Center and the University at Buffalo.

\section{Immunofluorescence staining and BrdU labeling}

Immunofluorescence staining was performed as previously described [75, 105107]. Briefly, tissues dissected from mice were fixed in $4 \%$ paraformaldehyde (PFA) in PBS for 30 min at $4^{\circ} \mathrm{C}$. Tissues were then rinsed with cold PBS $(\mathrm{pH} 7.4)$ plus $0.1 \%$ Tween 20 (PBST) three times for $20 \mathrm{~min}$ each, cryoprotected in $30 \%$ sucrose overnight, embedded, and frozen in OCT compound. The embedded tissues then were cut at $16 \mu \mathrm{m}$ thickness. The sections were washed three times for 10 min with PBST and blocked with 2\% BSA in PBST for 60 min and then were incubated with primary antibodies for $60 \mathrm{~min}$ at room temperature or overnight at $4{ }^{\circ} \mathrm{C}$. Then, sections were rinsed three times with PBST and incubated with fluorescent dye-conjugated secondary antibodies at room temperature for $60 \mathrm{~min}$. After sections were washed with PBST, they were nuclear counter-stained with propidium iodide $(\mathrm{PI})$ when necessary, and mounted with coverslips. Primary antibodies used included rat anti-BrdU (Abcam Ab6326, 1:200), goat anti-Pou4f2 (Brn3b) (Santa Cruz Sc-6026, 1:100), mouse anti-Pou4f1 (Brn3a) (Millipore Mab1585, 1:400), sheep anti-Vsx2 (Chx10) (Exalpha ABIN265011, 1:400), rabbit anti-cone arrestin (CAR, Millipore AB15282, 1:1000), goat anti-Calbindin-D28K (R\&D systems AF3320, 1:300), rabbit anti-Crx (Invitrogen PA5-111077, 1:200), rabbit anti-Caspase3 (R\&D systems AF835,1:200), goat anti-Isl1 (R\&D systems AF1837, 1:100), mouse anti-Nr2e3 (R\&D systems PP-H7223,1:100), goat anti-Nrl (R\&D systems AF2945, 1:200), rabbit anti-Otx2 (Sigma B74059, 1:200), mouse anti-Pax6 (DSHB AB_528427, 1:400), mouse anti-PCNA (Sigma P8825, 1:200), mouse anti-pH3 (Cell signaling 9706, 1:100), mouse anti-Rhodopsin (Sigma O4886, 1:400), rabbit anti-Sox9 (Millipore AB5535, 1:1000), mouse anti-Syntaxin (Sigma S0664, 1:200), mouse anti-Vimentin (Sigma V2258, 1:400), and rabbit antiZfp36l1/2 (Cell signaling 2119,1:100). Secondary antibodies were purchased from Life Sciences. 
BrdU labeling followed a procedure we have described previously by injecting pregnant mice at the desired stage intraperitoneally BrdU in PBS at $10 \mathrm{mg} / \mathrm{kg}$ body weight and harvesting the embryos one hour after injection [29]. They were further processed, embedded in OCT, and sectioned as described above. The sections were then treated with $4 \mathrm{~N} \mathrm{HCl}$ for 1.5 hours to expose the BrdU epitope, neutralized with $0.1 \mathrm{M}$ sodium borate ( $\mathrm{pH} 8.5)$, and immunofluorescence stained by an anti-BrdU antibody (Abcam Ab6326, 1:200).

\section{Confocal Imaging and Cell Counting}

Confocal fluorescence images were collected using a Leica TCS SP2 confocal microscope. Image contrast adjustment, when needed, was done identically for control and test specimens using Adobe Photoshop CS5. For cell counting on retinal sections, cells positive for specific markers from arbitrary unit lengths in the central regions were counted manually. At least three $(n=3)$ sections from different animals were counted, and a two-tailed, two-sample of equal variance Student's $t$-test was performed; $p<0.05$ was considered significant and $p<0.01$ highly significant.

\section{Hemotoxylin and Eosin (H\&E) Staining}

H\&E staining was carried out as previously described [35]. The tissues were washed with PBS, then fixed with buffered mixed aldehydes $(3 \%$ paraformaldehyde and $2 \%$ glutaraldehyde, in $\mathrm{PBS}, \mathrm{pH} 7.4$ ) for more than 16 hours at room temperature, dehydrated with $25 \%, 50 \%, 70 \%$, and $100 \%$ ethanol gradients for $40 \mathrm{~min}$ each at room temperature, again dehydrated with $100 \%$ ethanol at $4^{\circ} \mathrm{C}$ for overnight, and finally embedded in paraffin and sectioned. Sections of $7 \mu \mathrm{m}$ were baked for $1 \mathrm{~h}$ at $60^{\circ} \mathrm{C}$ in an oven, dewaxed in xylene twice for 5 min each, dehydrated in $100 \%$ ethanol twice for 2 min each, and stained with H\&E. Images were obtained with a Nikon Eclipse 80i microscope using a SPOT RT3 digital camera (Diagnostic Instruments).

\section{RNAscope in situ hybridization}

Probes and other reagents for RNAscope hybridization were purchased from Advanced Cell Diagnostics (ACD). The hybridization followed the manufacturer's instructions on paraffin retinal sections prepared as described above. The sections were dehydrated and dried and then treated with hydrogen peroxide solution for $10 \mathrm{~min}$ at room temperature and washed with distilled water, and followed by incubation with target retrieval reagent (Cat. No.322000) maintained at a boiling temperature using a hot plate for $15 \mathrm{~min}$ and additional washing with distilled water. The sections were then treated with Protease plus (Cat. No.322330) reagents for 30 min at $40^{\circ} \mathrm{C}$ in a HybEZ hybridization oven. They were then incubated with the RNAscope probes for $2 \mathrm{~h}$ at $40^{\circ} \mathrm{C}$ in a HybEZ hybridization oven. The slides were repeatedly washed twice with the wash buffer reagent (Cat. No.310091). Signal amplification and detection reagents (Cat. No. 322310) were applied sequentially and incubated in the order of AMP 1, AMP 2, AMP 3, AMP 4, AMP 5, and AMP 6, for $30,15,30,15,30,15 \mathrm{~min}$, respectively. Signal detection was carried out using 
the Fast Red detection system (RNAscope ${ }^{\circledR} 2.5$ HD Reagent Kit-RED) for $10 \mathrm{~min}$ at room temperature. The sections were then counterstained with $20 \%$ hematoxylin for 2 min, rinsed with tap water, placed in $0.02 \%$ ammonium water, followed by another tap water rinse. The sections were baked for $15 \mathrm{~min}$ at $60^{\circ} \mathrm{C}$ in an oven and mounted for imaging with a Nikon Eclipse 80i microscope.

\section{RNA-Seq}

After timed mating, E14.5, E17.5, and P0 retinas from control (Zfp36/1F/F;Zfp36/2F/F) and Zfp36/1/2 double knockout (DKO, Zfp36/1F/F;Zfp36/2F/F;Vsx2-Cre) mice were collected in ice-cold PBS and treated overnight with RNAlater solution (Ambion, AM7020) at $4{ }^{\circ} \mathrm{C}$, and finally stored at $-80^{\circ} \mathrm{C}$. Total RNA was isolated/purified using the miRNeasy Mini Kit (Qiagen, 217004) along with on-column digestion of DNA with RNase-Free DNase Set (Qiagen, 79254) following the manufacturers' instructions. Four independent samples were prepared for each genotype. RNA quality and quantity were assessed by a BioAnalyzer (Agilent, G2940CA). The samples were then used to generate sequencing libraries with TruSeq RNA Sample Prep Kit v2 kit (Illumina, RS-122-2001) and paired-end sequenced $(2 \times 75)$ on an Illumina Nextseq sequencer following the manufacturer's instructions.

The sequence reads were mapped to the mouse genome $(\mathrm{mm} 10)$ along with the GENCODE M20 using STAR v2.6.1d (https://www.ncbi.nlm.nih.gov/pubmed/23104886) with ENCODE Standard options. Gene expression quantification was performed using RSEM v1.3.1 [108] with parameters of paired-end, strandedness reverse, alignments, estimate-rspd, calc-ci, seed 12345, and ci-memory 30000. Differentially expressed genes (DEGs) were identified using DESeq2 by comparison between wild-type and mutant samples at the same developing stages (https://bioconductor.org/packages/release/bioc/html/DESeq2.html). Genes with an adjusted $p$ value of $\leqslant 0.05$ and fold change of $\geqslant 1.25$ were considered differentially expressed. Gene ontology analysis of the DEGs was performed using the DAVID web tool and the complete mouse genes were used as control. The sequence reads have all been deposited into the National Center for Biotechnology Information Gene Expression Omnibus (accession no GSE158718).

\section{In silico identification Zfp36/1 and Zfp36/2 target genes in the retina}

Two methods were used to achieve this. The first was to compare our DEG lists obtained by RNA-seq with a confirmed Zfp36l1 target list from the thymus [63]. Secondly, we examined the presence of AREs in the 3' UTRs of the DEGs. To do this, we used an R script to search for the presence of ARE motifs in the 3'UTR of the DEGs identified by RNA-seq. Although originally the ARE motif was defined as TATTTATT [90], adjacent TATT motifs closely spaced can also be bound by the TTP proteins efficiently [91]. Thus, both the TATT motif and the TATTTATT motif were searched. For genes with multiple mRNA isoforms, the one with the longest 3' UTR that covers the sequences in all the highest confidence isoforms (based on transcript support level and presence of a CCDS) was used. When this was not 
possible, additional isoforms were searched to ensure full coverage. Genes containing at least one TATTTATT motif or two adjacent TATT motifs separated by less than three bases were considered a positive hit. For genes that more than one isoforms were searched, only the one having the most TATTTATT motifs, or closest spaced TATT motifs, were reported. These analyses allowed us to identify potential target genes among the up- and downregulated DEGs for all the three stages examined.

To examine whether the potential targets identified in the DEG lists were enriched, we used a pipeline to generate 100 control gene sets matched with the DEG gene set of interest by expression level (FPKM) in thymus [63] and 3'UTR length. Each of these 100 sets was identical in size to the DEG set to be tested, i.e. each gene within the set has one matching gene in each control set. The number of targets in the gene set of interest, either based on comparison with the thymus iCLIP list or presence of ARE motifs, were compared to the numbers of targets within the control gene sets. For the 100 control gene sets, the median and 5th/95th percentiles of the number of targets per set were calculated. Target genes in the gene set of interest were considered enriched or depleted if their numbers fall out of the 5th/95th range $(P<0.1)$.

\section{Acknowledgment}

We thank other members of the Mu laboratory and members of the Department of Ophthalmology and the Developmental Genomics Group, University of Buffalo, for helpful discussions. We also would like to thank Hemabindu Chintala and Jennifer Daily for their technical contributions at the early stages of this project. Construction and sequencing of RNA-seq libraries were carried out at the Genomics and Bioinformatics Core of University at Buffalo. Research reported in this publication was supported by the National Eye Institute of the National Institutes of Health under Award Numbers R01EY029705 and R01EY020545 to X.M. and Biotechnology and Biological Sciences Research Council grant $\mathrm{BBS} / \mathrm{E} / \mathrm{B} / 000 \mathrm{C} 0428$ to M.T. The content is solely the responsibility of the authors and does not necessarily represent the official views of funding agencies. 


\section{References}

1. Hoon M, Okawa H, Della Santina L, Wong RO (2014) Functional architecture of the retina: development and disease. Prog Retin Eye Res 42:44-84. https://doi.org/10.1016/j.preteyeres.2014.06.003

2. Masland RH (2001) The fundamental plan of the retina. Nat Neurosci 4:877-86. https://doi.org/10.1038/nn0901-877

3. Wassle H, Boycott BB (1991) Functional architecture of the mammalian retina. Physiol Rev 71:447-80. https://doi.org/10.1152/physrev.1991.71.2.447

4. Bringmann A, Pannicke T, Grosche J, et al (2006) Muller cells in the healthy and diseased retina. Prog Retin Eye Res 25:397-424. https://doi.org/10.1016/j.preteyeres.2006.05.003

5. Reichenbach A, Bringmann A (2013) New functions of Muller cells. Glia 61:651-78. https://doi.org/10.1002/glia.22477

6. Turner DL, Cepko CL (1987) A common progenitor for neurons and glia persists in rat retina late in development. Nature 328:131-6. https://doi.org/10.1038/328131a0

7. Turner DL, Snyder EY, Cepko CL (1990) Lineage-independent determination of cell type in the embryonic mouse retina. Neuron 4:833-45

8. Young RW (1985) Cell differentiation in the retina of the mouse. Anat Rec 212:199-205. https://doi.org/10.1002/ar.1092120215

9. Diacou R, Zhao Y, Zheng D, et al (2018) Six3 and Six6 Are Jointly Required for the Maintenance of Multipotent Retinal Progenitors through Both Positive and Negative Regulation. Cell Rep 25:2510-2523.e4. https://doi.org/10.1016/j.celrep.2018.10.106

10. Gordon PJ, Yun S, Clark AM, et al (2013) Lhx2 balances progenitor maintenance with neurogenic output and promotes competence state progression in the developing retina. J Neurosci 33:12197-207. https://doi.org/10.1523/JNEUROSCI.1494-13.2013

11. Green ES, Stubbs JL, Levine EM (2003) Genetic rescue of cell number in a mouse model of microphthalmia: interactions between $\mathrm{Chx10}$ and G1-phase cell cycle regulators. Development 130:539-52

12. Jadhav AP, Mason HA, Cepko CL (2006) Notch 1 inhibits photoreceptor production in the developing mammalian retina. Development 133:913-23. https://doi.org/10.1242/dev.02245

13. Jadhav AP, Cho SH, Cepko CL (2006) Notch activity permits retinal cells to progress through multiple progenitor states and acquire a stem cell property. Proc Natl Acad Sci U A 103:18998-9003. https://doi.org/10.1073/pnas.0608155103 
14. Marquardt T, Ashery-Padan R, Andrejewski N, et al (2001) Pax6 is required for the multipotent state of retinal progenitor cells. Cell 105:43-55

15. Mathers PH, Grinberg A, Mahon KA, Jamrich M (1997) The Rx homeobox gene is essential for vertebrate eye development. Nature 387:603-7. https://doi.org/10.1038/42475

16. de Melo J, Zibetti C, Clark BS, et al (2016) Lhx2 Is an Essential Factor for Retinal Gliogenesis and Notch Signaling. J Neurosci 36:2391-405.

https://doi.org/10.1523/JNEUROSCI.3145-15.2016

17. Taranova OV, Magness ST, Fagan BM, et al (2006) SOX2 is a dose-dependent regulator of retinal neural progenitor competence. Genes Dev 20:1187-202. https://doi.org/10.1101/gad.1407906

18. Yaron O, Farhy C, Marquardt T, et al (2006) Notch1 functions to suppress conephotoreceptor fate specification in the developing mouse retina. Development 133:1367-78. https://doi.org/10.1242/dev.02311

19. Austin CP, Feldman DE, Ida JA Jr, Cepko CL (1995) Vertebrate retinal ganglion cells are selected from competent progenitors by the action of Notch. Development 121:3637-50

20. Furukawa T, Mukherjee S, Bao ZZ, et al (2000) rax, Hes1, and notch1 promote the formation of Muller glia by postnatal retinal progenitor cells. Neuron 26:383-94

21. Kong JH, Yang L, Dessaud E, et al (2015) Notch activity modulates the responsiveness of neural progenitors to sonic hedgehog signaling. Dev Cell 33:373-87. https://doi.org/10.1016/j.devcel.2015.03.005

22. Maurer KA, Riesenberg AN, Brown NL (2014) Notch signaling differentially regulates Atoh7 and Neurog2 in the distal mouse retina. Development 141:3243-54. https://doi.org/10.1242/dev.106245

23. Nelson BR, Gumuscu B, Hartman BH, Reh TA (2006) Notch activity is downregulated just prior to retinal ganglion cell differentiation. Dev Neurosci 28:128-41. https://doi.org/10.1159/000090759

24. Perron M, Harris WA (2000) Determination of vertebrate retinal progenitor cell fate by the Notch pathway and basic helix-loop-helix transcription factors. Cell Mol Life Sci 57:215-23. https://doi.org/10.1007/PL00000685

25. Riesenberg AN, Brown NL (2016) Cell autonomous and nonautonomous requirements for Delltalike1 during early mouse retinal neurogenesis. Dev Dyn Off Publ Am Assoc Anat 245:631-640. https://doi.org/10.1002/dvdy.24402

26. Hashimoto T, Zhang XM, Chen BY, Yang XJ (2006) VEGF activates divergent intracellular signaling components to regulate retinal progenitor cell proliferation and neuronal differentiation. Development 133:2201-10. https://doi.org/10.1242/dev.02385 
27. Kim J, Wu HH, Lander AD, et al (2005) GDF11 controls the timing of progenitor cell competence in developing retina. Science 308:1927-30. https://doi.org/10.1126/science.1110175

28. Mu X, Beremand PD, Zhao S, et al (2004) Discrete gene sets depend on POU domain transcription factor Brn3b/Brn-3.2/POU4f2 for their expression in the mouse embryonic retina. Development 131:1197-210. https://doi.org/10.1242/dev.01010

29. Mu X, Fu X, Sun H, et al (2005) Ganglion cells are required for normal progenitor- cell proliferation but not cell-fate determination or patterning in the developing mouse retina. Curr Biol 15:525-30. https://doi.org/10.1016/j.cub.2005.01.043

30. Wang Y, Dakubo GD, Thurig S, et al (2005) Retinal ganglion cell-derived sonic hedgehog locally controls proliferation and the timing of RGC development in the embryonic mouse retina. Development 132:5103-13. https://doi.org/10.1242/dev.02096

31. Zhang XM, Yang XJ (2001) Regulation of retinal ganglion cell production by Sonic hedgehog. Development 128:943-57

32. Brown NL, Patel S, Brzezinski J, Glaser T (2001) Math5 is required for retinal ganglion cell and optic nerve formation. Development 128:2497-508

33. Gan L, Xiang M, Zhou L, et al (1996) POU domain factor Brn-3b is required for the development of a large set of retinal ganglion cells. Proc Natl Acad Sci U A 93:3920-5

34. Jiang $Y$, Ding $Q$, Xie X, et al (2013) Transcription factors SOX4 and SOX11 function redundantly to regulate the development of mouse retinal ganglion cells. J Biol Chem 288:18429-38. https://doi.org/10.1074/jbc.M113.478503

35. Mu X, Fu X, Beremand PD, et al (2008) Gene regulation logic in retinal ganglion cell development: Isl1 defines a critical branch distinct from but overlapping with Pou4f 2 . Proc Natl Acad Sci U A 105:6942-7. https://doi.org/10.1073/pnas.0802627105

36. Wang SW, Kim BS, Ding K, et al (2001) Requirement for math5 in the development of retinal ganglion cells. Genes Dev 15:24-9

37. Dyer MA, Livesey FJ, Cepko CL, Oliver G (2003) Prox1 function controls progenitor cell proliferation and horizontal cell genesis in the mammalian retina. Nat Genet 34:53-8. https://doi.org/10.1038/ng1144

38. Fujitani Y, Fujitani S, Luo H, et al (2006) Ptf1a determines horizontal and amacrine cell fates during mouse retinal development. Development 133:4439-50.

https://doi.org/10.1242/dev.02598

39. Hicks EA, Zaveri M, Deschamps PA, et al (2018) Conditional Deletion of AP-2 $\alpha$ and AP-2 $\beta$ in the Developing Murine Retina Leads to Altered Amacrine Cell Mosaics and Disrupted Visual Function. Invest Ophthalmol Vis Sci 59:2229-2239.

https://doi.org/10.1167/iovs.17-23283 
40. Jin K, Jiang H, Xiao D, et al (2015) Tfap2a and $2 \mathrm{~b}$ act downstream of Ptf1a to promote amacrine cell differentiation during retinogenesis. Mol Brain 8:28.

https://doi.org/10.1186/s13041-015-0118-x

41. Li S, Mo Z, Yang X, et al (2004) Foxn4 controls the genesis of amacrine and horizontal cells by retinal progenitors. Neuron 43:795-807.

https://doi.org/10.1016/j.neuron.2004.08.041

42. Chen S, Wang Q-L, Xu S, et al (2002) Functional analysis of cone-rod homeobox (CRX) mutations associated with retinal dystrophy. Hum Mol Genet 11:873-884. https://doi.org/10.1093/hmg/11.8.873

43. Furukawa T, Morrow EM, Li T, et al (1999) Retinopathy and attenuated circadian entrainment in Crx-deficient mice. Nat Genet 23:466-70. https://doi.org/10.1038/70591

44. Mears AJ, Kondo M, Swain PK, et al (2001) Nrl is required for rod photoreceptor development. Nat Genet 29:447-52. https://doi.org/10.1038/ng774

45. Nishida A, Furukawa A, Koike C, et al (2003) Otx2 homeobox gene controls retinal photoreceptor cell fate and pineal gland development. Nat Neurosci 6:1255-63. https://doi.org/10.1038/nn1155

46. Peng GH, Ahmad O, Ahmad F, et al (2005) The photoreceptor-specific nuclear receptor $\mathrm{Nr} 2 \mathrm{e} 3$ interacts with $\mathrm{Crx}$ and exerts opposing effects on the transcription of rod versus cone genes. Hum Mol Genet 14:747-64. https://doi.org/10.1093/hmg/ddi070

47. Pennesi ME, Cho JH, Yang Z, et al (2003) BETA2/NeuroD1 null mice: a new model for transcription factor-dependent photoreceptor degeneration. J Neurosci 23:453-61

48. Carpenter S, Ricci EP, Mercier BC, et al (2014) Post-transcriptional regulation of gene expression in innate immunity. Nat Rev Immunol 14:361-76.

https://doi.org/10.1038/nri3682

49. Sanchez-Diaz P, Penalva LO (2006) Post-transcription meets post-genomic: the saga of RNA binding proteins in a new era. RNA Biol 3:101-9

50. Weil TT (2015) Post-transcriptional regulation of early embryogenesis. F1000Prime Rep 7:31. https://doi.org/10.12703/P7-31

51. Blackshear PJ, Perera L (2014) Phylogenetic distribution and evolution of the linked RNAbinding and NOT1-binding domains in the tristetraprolin family of tandem CCCH zinc finger proteins. J Interferon Cytokine Res 34:297-306.

https://doi.org/10.1089/jir.2013.0150

52. Ma W, Mayr C (2018) A Membraneless Organelle Associated with the Endoplasmic Reticulum Enables 3'UTR-Mediated Protein-Protein Interactions. Cell 175:1492-1506.e19. https://doi.org/10.1016/j.cell.2018.10.007 
53. Moore MJ, Blachere NE, Fak JJ, et al (2018) ZFP36 RNA-binding proteins restrain T cell activation and anti-viral immunity. eLife 7:. https://doi.org/10.7554/eLife.33057

54. Pereira B, Billaud M, Almeida R (2017) RNA-Binding Proteins in Cancer: Old Players and New Actors. Trends Cancer 3:506-528. https://doi.org/10.1016/j.trecan.2017.05.003

55. Perez-Ortin JE, Alepuz P, Chavez S, Choder M (2013) Eukaryotic mRNA decay: methodologies, pathways, and links to other stages of gene expression. J Mol Biol 425:3750-75. https://doi.org/10.1016/j.jmb.2013.02.029

56. Sanduja S, Blanco FF, Dixon DA (2011) The roles of TTP and BRF proteins in regulated mRNA decay. Wiley Interdiscip Rev RNA 2:42-57. https://doi.org/10.1002/wrna.28

57. Brooks SA, Blackshear PJ (2013) Tristetraprolin (TTP): interactions with mRNA and proteins, and current thoughts on mechanisms of action. Biochim Biophys Acta 1829:666-79. https://doi.org/10.1016/j.bbagrm.2013.02.003

58. Tiedje C, Diaz-Munoz MD, Trulley P, et al (2016) The RNA-binding protein TTP is a global post-transcriptional regulator of feedback control in inflammation. Nucleic Acids Res 44:7418-40. https://doi.org/10.1093/nar/gkw474

59. Wells ML, Hicks SN, Perera L, Blackshear PJ (2015) Functional equivalence of an evolutionarily conserved RNA binding module. J Biol Chem 290:24413-23. https://doi.org/10.1074/jbc.M115.673012

60. Bye-A-Jee $H$, Pugazhendhi $D$, Woodhouse $S$, et al (2018) The RNA-binding proteins Zfp36l1 and Zfp36l2 act redundantly in myogenesis. Skelet Muscle 8:37. https://doi.org/10.1186/s13395-018-0183-9

61. Galloway A, Saveliev A, Lukasiak S, et al (2016) RNA-binding proteins ZFP36L1 and ZFP36L2 promote cell quiescence. Science 352:453-9.

https://doi.org/10.1126/science.aad5978

62. Hodson DJ, Janas ML, Galloway A, et al (2010) Deletion of the RNA-binding proteins ZFP36L1 and ZFP36L2 leads to perturbed thymic development and T lymphoblastic leukemia. Nat Immunol 11:717-24. https://doi.org/10.1038/ni.1901

63. Vogel KU, Bell LS, Galloway A, et al (2016) The RNA-Binding Proteins Zfp36I1 and Zfp36/2 Enforce the Thymic beta-Selection Checkpoint by Limiting DNA Damage Response Signaling and Cell Cycle Progression. J Immunol 197:2673-2685. https://doi.org/10.4049/jimmunol.1600854

64. Alvarez-Bolado G, Eichele $\mathrm{G}$ (2006) Analysing the developing brain transcriptome with the GenePaint platform. J Physiol 575:347-52. https://doi.org/10.1113/jphysiol.2006.112763

65. Cargnin F, Nechiporuk T, Mullendorff K, et al (2014) An RNA binding protein promotes axonal integrity in peripheral neurons by destabilizing REST. J Neurosci 34:16650-61. https://doi.org/10.1523/JNEUROSCI.1650-14.2014 
66. Dai W, Li W, Hoque M, et al (2015) A post-transcriptional mechanism pacing expression of neural genes with precursor cell differentiation status. Nat Commun 6:7576. https://doi.org/10.1038/ncomms8576

67. Wu F, Bard JE, Kann J, et al (2020) Single cell transcriptomics reveals lineage trajectory of the retinal ganglion cells in wild-type and Atoh7-null retinas. bioRxiv 2020.02.26.966093. https://doi.org/10.1101/2020.02.26.966093

68. Bell SE, Sanchez MJ, Spasic-Boskovic O, et al (2006) The RNA binding protein Zfp36l1 is required for normal vascularisation and post-transcriptionally regulates VEGF expression. Dev Dyn 235:3144-55. https://doi.org/10.1002/dvdy.20949

69. Ramos SB, Stumpo DJ, Kennington EA, et al (2004) The CCCH tandem zinc-finger protein Zfp36l2 is crucial for female fertility and early embryonic development. Development 131:4883-93. https://doi.org/10.1242/dev.01336

70. Stumpo DJ, Byrd NA, Phillips RS, et al (2004) Chorioallantoic fusion defects and embryonic lethality resulting from disruption of $\mathrm{Zfp} 36 \mathrm{~L} 1$, a gene encoding a $\mathrm{CCCH}$ tandem zinc finger protein of the Tristetraprolin family. Mol Cell Biol 24:6445-55.

https://doi.org/10.1128/MCB.24.14.6445-6455.2004

71. Stumpo DJ, Broxmeyer HE, Ward T, et al (2009) Targeted disruption of Zfp36/2, encoding a CCCH tandem zinc finger RNA-binding protein, results in defective hematopoiesis. Blood 114:2401-10. https://doi.org/10.1182/blood-2009-04-214619

72. Furuta $\mathrm{Y}$, Behringer RR (2005) Recent innovations in tissue-specific gene modifications in the mouse. Birth Defects Res C Embryo Today 75:43-57.

https://doi.org/10.1002/bdrc.20036

73. Rowan S, Cepko CL (2004) Genetic analysis of the homeodomain transcription factor $\mathrm{Ch} \times 10$ in the retina using a novel multifunctional BAC transgenic mouse reporter. Dev Biol 271:388-402. https://doi.org/10.1016/j.ydbio.2004.03.039

74. Fu X, Sun H, Klein WH, MuX (2006) Beta-catenin is essential for lamination but not neurogenesis in mouse retinal development. Dev Biol 299:424-37. https://doi.org/10.1016/j.ydbio.2006.08.015

75. Wu F, Kaczynski TJ, Sethuramanujam S, et al (2015) Two transcription factors, Pou4f2 and Isl1, are sufficient to specify the retinal ganglion cell fate. Proc Natl Acad Sci U A 112:E1559-68. https://doi.org/10.1073/pnas.1421535112

76. Geissler R, Grimson A (2016) A position-specific 3'UTR sequence that accelerates mRNA decay. RNA Biol 13:1075-1077. https://doi.org/10.1080/15476286.2016.1225645

77. Grimson A, Farh KK-H, Johnston WK, et al (2007) MicroRNA targeting specificity in mammals: determinants beyond seed pairing. Mol Cell 27:91-105. https://doi.org/10.1016/j.molcel.2007.06.017 
78. Huang DW, Sherman BT, Lempicki RA (2009) Bioinformatics enrichment tools: paths toward the comprehensive functional analysis of large gene lists. Nucleic Acids Res 37:113. https://doi.org/10.1093/nar/gkn923

79. Bosanac I, Maun HR, Scales SJ, et al (2009) The structure of SHH in complex with HHIP reveals a recognition role for the Shh pseudo active site in signaling. Nat Struct Mol Biol 16:691-7. https://doi.org/10.1038/nsmb.1632

80. Huang $\mathrm{H}-\mathrm{Y}$, Chien $\mathrm{C}-\mathrm{H}$, Jen $\mathrm{K}-\mathrm{H}$, Huang H-D (2006) RegRNA: an integrated web server for identifying regulatory RNA motifs and elements. Nucleic Acids Res 34:W429-434. https://doi.org/10.1093/nar/gkl333

81. Lelievre EC, Lek M, Boije $\mathrm{H}$, et al (2011) Ptf1a/Rbpj complex inhibits ganglion cell fate and drives the specification of all horizontal cell subtypes in the chick retina. Dev Biol 358:296-308. https://doi.org/10.1016/j.ydbio.2011.07.033

82. Luo H, Jin K, Xie Z, et al (2012) Forkhead box N4 (Foxn4) activates Dll4-Notch signaling to suppress photoreceptor cell fates of early retinal progenitors. Proc Natl Acad Sci U S A 109:E553-562. https://doi.org/10.1073/pnas.1115767109

83. Mizeracka K, DeMaso CR, Cepko CL (2013) Notch1 is required in newly postmitotic cells to inhibit the rod photoreceptor fate. Development 140:3188-97.

https://doi.org/10.1242/dev.090696

84. Riesenberg AN, Liu Z, Kopan R, Brown NL (2009) Rbpj cell autonomous regulation of retinal ganglion cell and cone photoreceptor fates in the mouse retina. J Neurosci 29:12865-77. https://doi.org/10.1523/JNEUROSCI.3382-09.2009

85. Nelson BR, Hartman BH, Georgi SA, et al (2007) Transient inactivation of Notch signaling synchronizes differentiation of neural progenitor cells. Dev Biol 304:479-498. https://doi.org/10.1016/j.ydbio.2007.01.001

86. Sakagami K, Gan L, Yang X-J (2009) Distinct effects of Hedgehog signaling on neuronal fate specification and cell cycle progression in the embryonic mouse retina. J Neurosci Off J Soc Neurosci 29:6932-6944. https://doi.org/10.1523/JNEUROSCI.0289-09.2009

87. Wall DS, Mears AJ, McNeill B, et al (2009) Progenitor cell proliferation in the retina is dependent on Notch-independent Sonic hedgehog/Hes1 activity. J Cell Biol 184:101-12. https://doi.org/10.1083/jcb.200805155

88. Ciais D, Cherradi N, Bailly S, et al (2004) Destabilization of vascular endothelial growth factor mRNA by the zinc-finger protein TIS11b. Oncogene 23:8673-8680. https://doi.org/10.1038/sj.onc.1207939

89. Planel $S$, Salomon A, Jalinot $P$, et al (2010) A novel concept in antiangiogenic and antitumoral therapy: multitarget destabilization of short-lived mRNAs by the zinc finger protein ZFP36L1. Oncogene 29:5989-6003. https://doi.org/10.1038/onc.2010.341 
90. Hudson BP, Martinez-Yamout MA, Dyson HJ, Wright PE (2004) Recognition of the mRNA AU-rich element by the zinc finger domain of TIS11d. Nat Struct Mol Biol 11:257-264. https://doi.org/10.1038/nsmb738

91. Mukherjee N, Jacobs NC, Hafner M, et al (2014) Global target mRNA specification and regulation by the RNA-binding protein ZFP36. Genome Biol 15:R12. https://doi.org/10.1186/gb-2014-15-1-r12

92. Qi X, Li X (2020) Mechanistic Insights into the Generation and Transduction of Hedgehog Signaling. Trends Biochem Sci 45:397-410. https://doi.org/10.1016/j.tibs.2020.01.006

93. Varjosalo M, Taipale J (2008) Hedgehog: functions and mechanisms. Genes Dev 22:24542472. https://doi.org/10.1101/gad.1693608

94. Dyer MA, Cepko CL (2000) p57(Kip2) regulates progenitor cell proliferation and amacrine interneuron development in the mouse retina. Development 127:3593-605

95. Chen MT, Dong L, Zhang XH, et al (2015) ZFP36L1 promotes monocyte/macrophage differentiation by repressing CDK6. Sci Rep 5:16229. https://doi.org/10.1038/srep16229

96. Zekavati A, Nasir A, Alcaraz A, et al (2014) Post-transcriptional regulation of BCL2 mRNA by the RNA-binding protein ZFP36L1 in malignant B cells. PLoS One 9:e102625. https://doi.org/10.1371/journal.pone.0102625

97. Liu J, Lu W, Liu S, et al (2018) ZFP36L2, a novel AML1 target gene, induces AML cells apoptosis and inhibits cell proliferation. Leuk Res 68:15-21. https://doi.org/10.1016/j.leukres.2018.02.017

98. Loh X-Y, Sun Q-Y, Ding L-W, et al (2020) RNA-Binding Protein ZFP36L1 Suppresses Hypoxia and Cell-Cycle Signaling. Cancer Res 80:219-233. https://doi.org/10.1158/00085472.CAN-18-2796

99. Suk F-M, Chang C-C, Lin R-J, et al (2018) ZFP36L1 and ZFP36L2 inhibit cell proliferation in a cyclin D-dependent and p53-independent manner. Sci Rep 8:2742. https://doi.org/10.1038/s41598-018-21160-z

100. Sneezum L, Eislmayr K, Dworak H, et al (2020) Context-Dependent IL-1 mRNADestabilization by TTP Prevents Dysregulation of Immune Homeostasis Under Steady State Conditions. Front Immunol 11:1398. https://doi.org/10.3389/fimmu.2020.01398

101. Adachi S, Homoto M, Tanaka R, et al (2014) ZFP36L1 and ZFP36L2 control LDLR mRNA stability via the ERK-RSK pathway. Nucleic Acids Res 42:10037-49. https://doi.org/10.1093/nar/gku652

102. Kondo M, Noguchi A, Matsuura Y, et al (2018) Novel phosphorelay-dependent control of ZFP36L1 protein during the cell cycle. Biochem Biophys Res Commun 501:387-393. https://doi.org/10.1016/j.bbrc.2018.04.212 
103. Lu H, Tran L, Park Y, et al (2018) Reciprocal Regulation of DUSP9 and DUSP16 Expression by HIF1 Controls ERK and p38 MAP Kinase Activity and Mediates Chemotherapy-Induced Breast Cancer Stem Cell Enrichment. Cancer Res 78:4191-4202. https://doi.org/10.1158/0008-5472.CAN-18-0270

104. Furuta Y, Lagutin O, Hogan BL, Oliver GC (2000) Retina- and ventral forebrain-specific Cre recombinase activity in transgenic mice. Genesis 26:130-2

105. Sapkota D, Chintala H, Wu F, et al (2014) Onecut1 and Onecut2 redundantly regulate early retinal cell fates during development. Proc Natl Acad Sci U A 111:E4086-4095. https://doi.org/10.1073/pnas.1405354111

106. Wu F, Sapkota D, Li R, MuX (2012) Onecut 1 and Onecut 2 are potential regulators of mouse retinal development. J Comp Neurol 520:952-69. https://doi.org/10.1002/cne.22741

107. Wu F, Li R, Umino Y, et al (2013) Onecut1 is essential for horizontal cell genesis and retinal integrity. J Neurosci 33:13053-65, 13065a. https://doi.org/10.1523/JNEUROSCI.0116-13.2013

108. Li B, Dewey CN (2011) RSEM: accurate transcript quantification from RNA-Seq data with or without a reference genome. BMC Bioinformatics 12:323.

https://doi.org/10.1186/1471-2105-12-323 

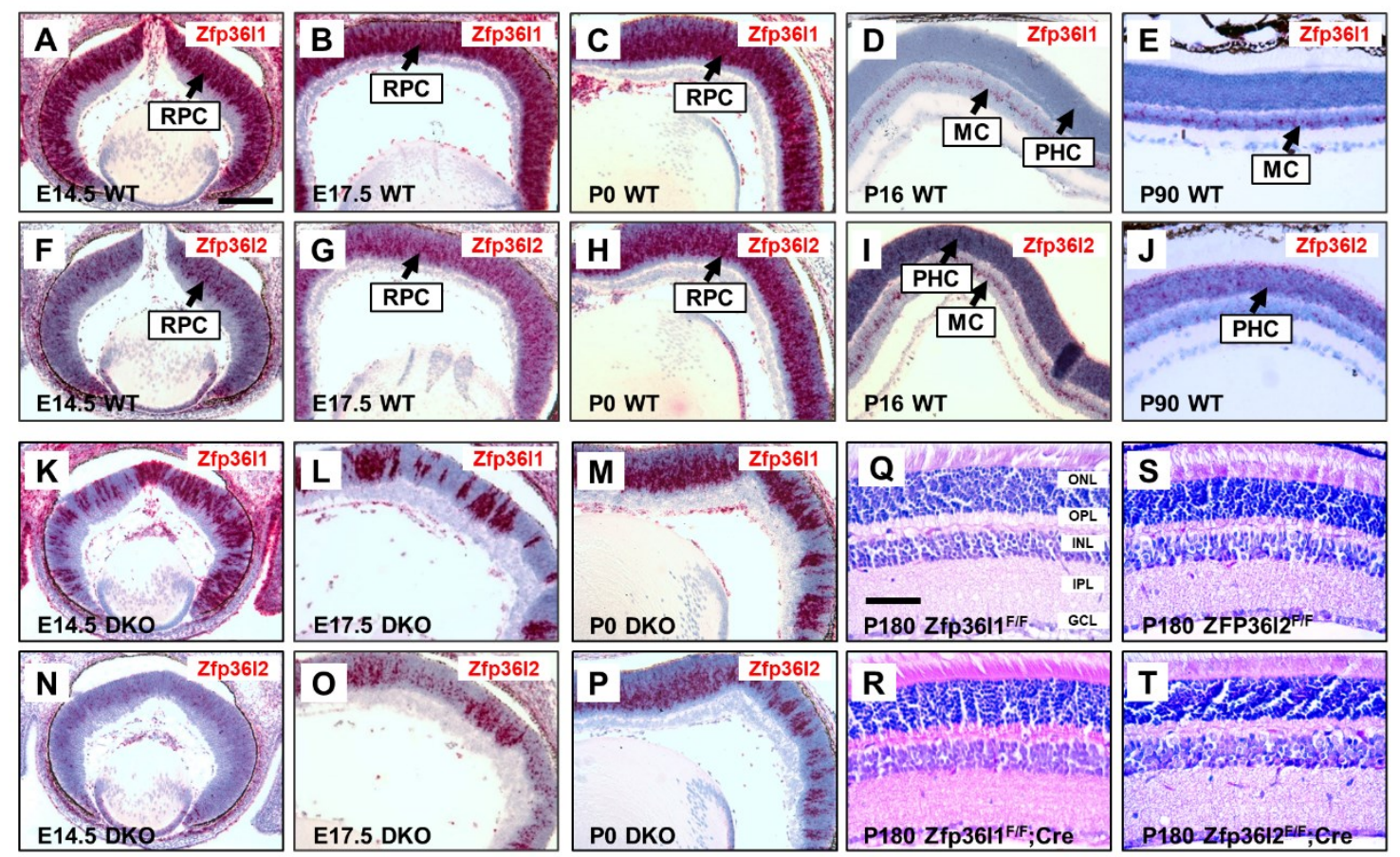

Figure 1. Expression and conditional deletion of Zfp36/1 and Zfp36/2 in the retina. A-J. RNAscope in situ hybridization on wild-type retinas (WT) shows that Zfp36/1 and Zfp36/2 have dynamic and similar expression patterns during retinal development. Red staining is in situ signals and blue is counterstaining by hematoxylin. At E14.5 (A, F), E17.5 (B, G), and P0 (C, H), both Zfp36/1 and Zfp36/2 are expressed in retinal progenitor cells (RPCs). At P16 and P90, Zfp36/1 is expressed weakly in photoreceptors (PHC) and strongly in Müller cells (MC) (D, E), whereas Zfp36/2 is expressed strongly in photoreceptors at both P16 and P90 and in Müller cells only at P16 (I, J). K-P. RNA-scope in situ hybridization reveals that Vsx2-Cre deletes Zfp36/1 and Zfp36/2 in a mosaic fashion at three different stages (E14.5, E17.5, and P0) of retinal development. Sections are from Zfp36/1F/F;Zfp36/2F/F;Vsx2-Cre (labeled as DKO) retinas. Note that the proportions of mutant cells were much reduced at PO (M, P). Q-T. H\&E staining showing single deletion of Zfp36/1 (R) and Zfp36/2 (T) by Six3-Cre does not lead to overt morphological defects at six months of age as compared to the controls $(\mathbf{Q}, \mathbf{S})$. ONL: outer nuclear layer; OPL: outer plexiform layer; INL: inner nuclear layer; IPL: inner plexiform layer; GCL: ganglion cell layer. The scale bar in $\mathbf{A}$ is $150 \mu \mathrm{m}$ and applies to panels A-P, and the scale bar in $\mathbf{Q}$ is $75 \mu \mathrm{m}$ and applies to panels $\mathbf{Q}-\mathbf{T}$. 

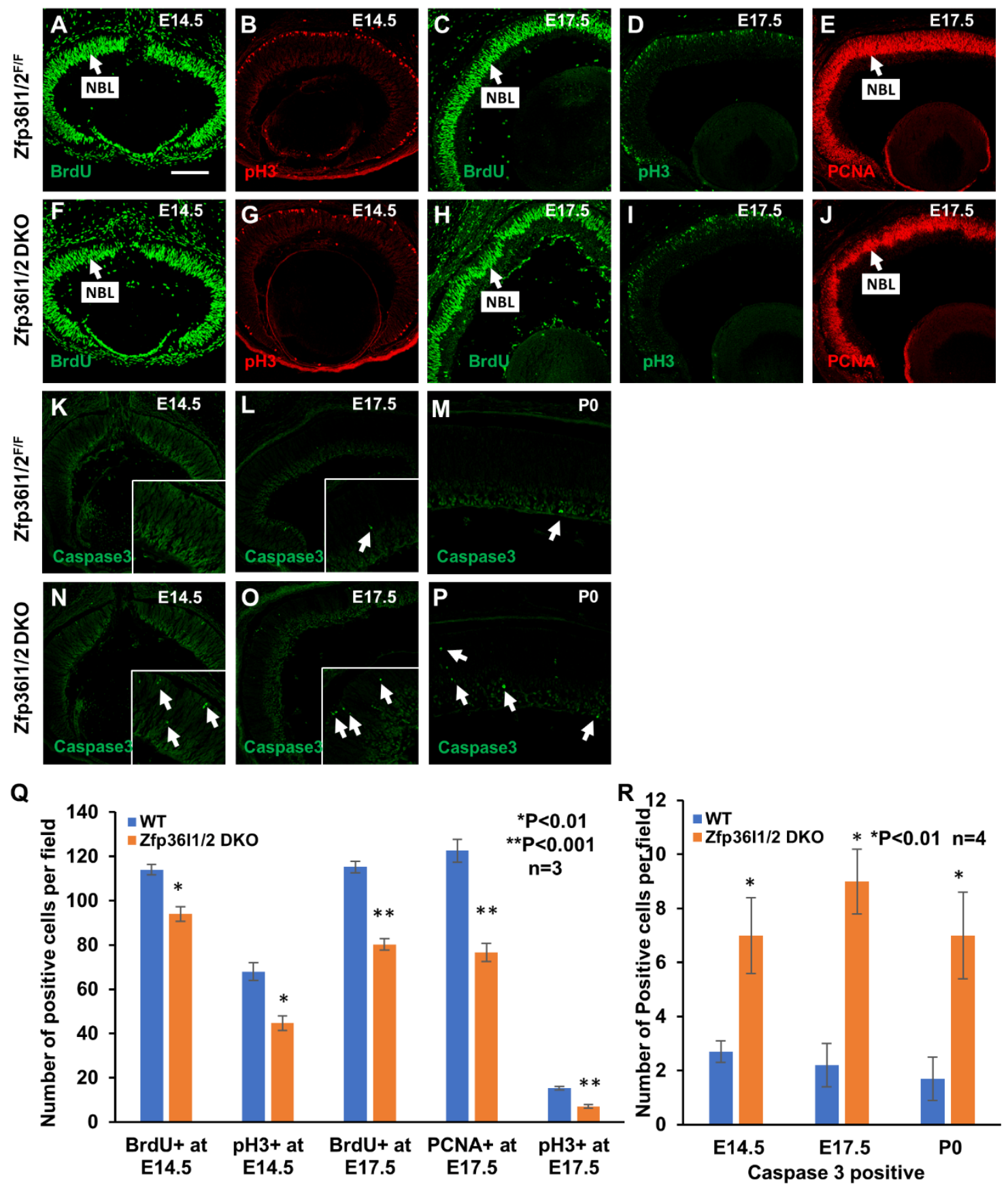

Figure 2. Double knockout of Zfp36/1 and Zfp36/2 by Vsx2-Cre leads to decreased proliferation and increased apoptosis. A, B, F, G. Immunostaining for $\mathrm{BrdU}(\mathbf{A}$ and $\mathbf{F}$ ) and $\mathrm{pH} 3$ ( $\mathbf{B}$ and $\mathbf{G}$ ) to label $\mathbf{S}$ and $\mathrm{M}$ phase RPCs respectively on E14.5 control (A, B) and Zfp36/1/2 DKO (F, G) retinal sections. C-E, H-J. Staining for BrdU ( $\mathbf{C}$ and $\mathbf{H}), \mathrm{pH} 3$ ( $\mathbf{D}$ and $\mathbf{I})$, and PCNA (E and $\mathbf{J})$ on E17.5 control (C-E) and Zfp36/1/2 DKO (H-J) retinal sections. Note the uneven thickness of the neuroblast layer $(\mathrm{NBL})$ in the DKO retina $(\mathbf{H}, \mathbf{J})$. K-P. Immunostaining for activated 
caspase3 on control (K-M) and Zfp36/1/2 DKO (N-P) sections from E14.5 (K and $\mathbf{N}$ ), E17.5 ( $\mathbf{L}$ and $\mathbf{O}$ ), and $P 0$ ( $\mathbf{M}$ and $\mathbf{P}$ ) retinas. Insets in $\mathbf{K}, \mathbf{L}, \mathbf{N}$, and $\mathbf{O}$ are enlarged areas of the corresponding sections to better show the apoptotic cells (arrows). Q. Quantitation of positive cells in A-J. The significance of differences between control and DKO retina was determined by Student's $t$ test. Error bars indicate \pm SD. R. Quantification of positive cells in K-P. The significance of differences between control and DKO retina was determined by Student's $t$ test. Error bars indicate \pm SD. The scale bar in $\mathbf{A}$ is $150 \mu \mathrm{m}$ and applies to all image panels. 

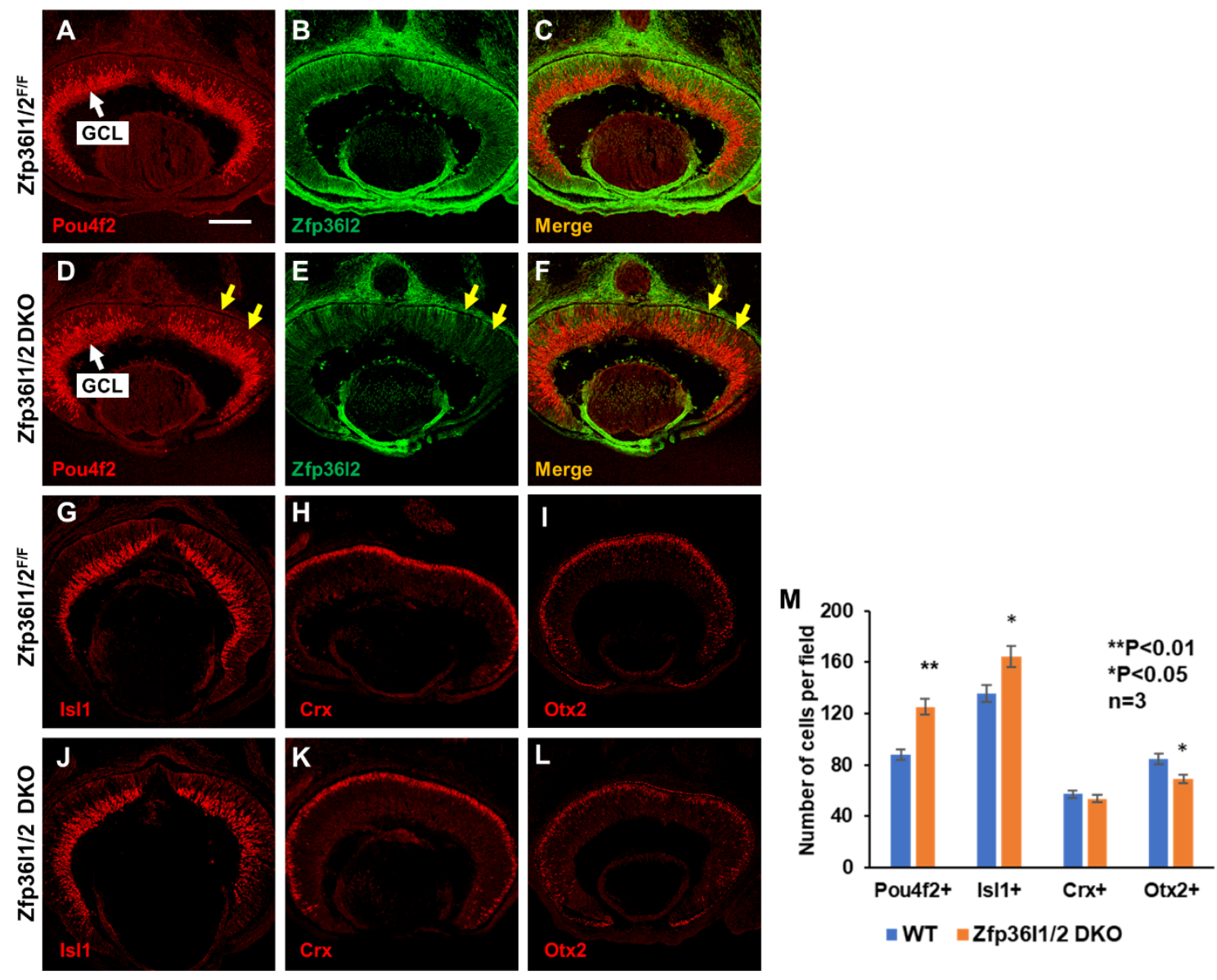

Figure 3. Double knockout of Zfp36/1 and Zfp36/2 increases RGCs, but not photoreceptors, at E14.5. A-F. Co-immunostaining for Pou4f2 (red) and Zfp36l1/2 (green) on E14.5 control (A-C) and Zfp36/1/2 DKO (D-F) retinal sections. Note the mosaic deletion pattern by Vsx2-Cre as revealed by anti-Zfp36|1/2 staining (E, F). Increased RGC genesis occurs more prominently in regions of deletion (yellow arrows) in the DKO retina. $\mathbf{G}$ and J. Staining for IsI 1 (red) on E14.5 control (G) and Zfp36/1/2 DKO (I) retinal sections. H-L. Staining for $\mathrm{Crx}(\mathbf{H}, \mathbf{K})$ and Otx2 (I, L), two photoreceptor markers, on E14.5 control $(\mathbf{H}, \mathbf{I})$ and DKO $(\mathbf{K}, \mathbf{L})$ retinal sections. M. Quantitation of positive cells in A, D, and G-L. Significance of differences (compared with WT controls) was determined by Student's $t$-test. Error bars indicate \pm SD. The scale bar in $\mathbf{A}$ is $150 \mu \mathrm{m}$ and applies to all image panels. 

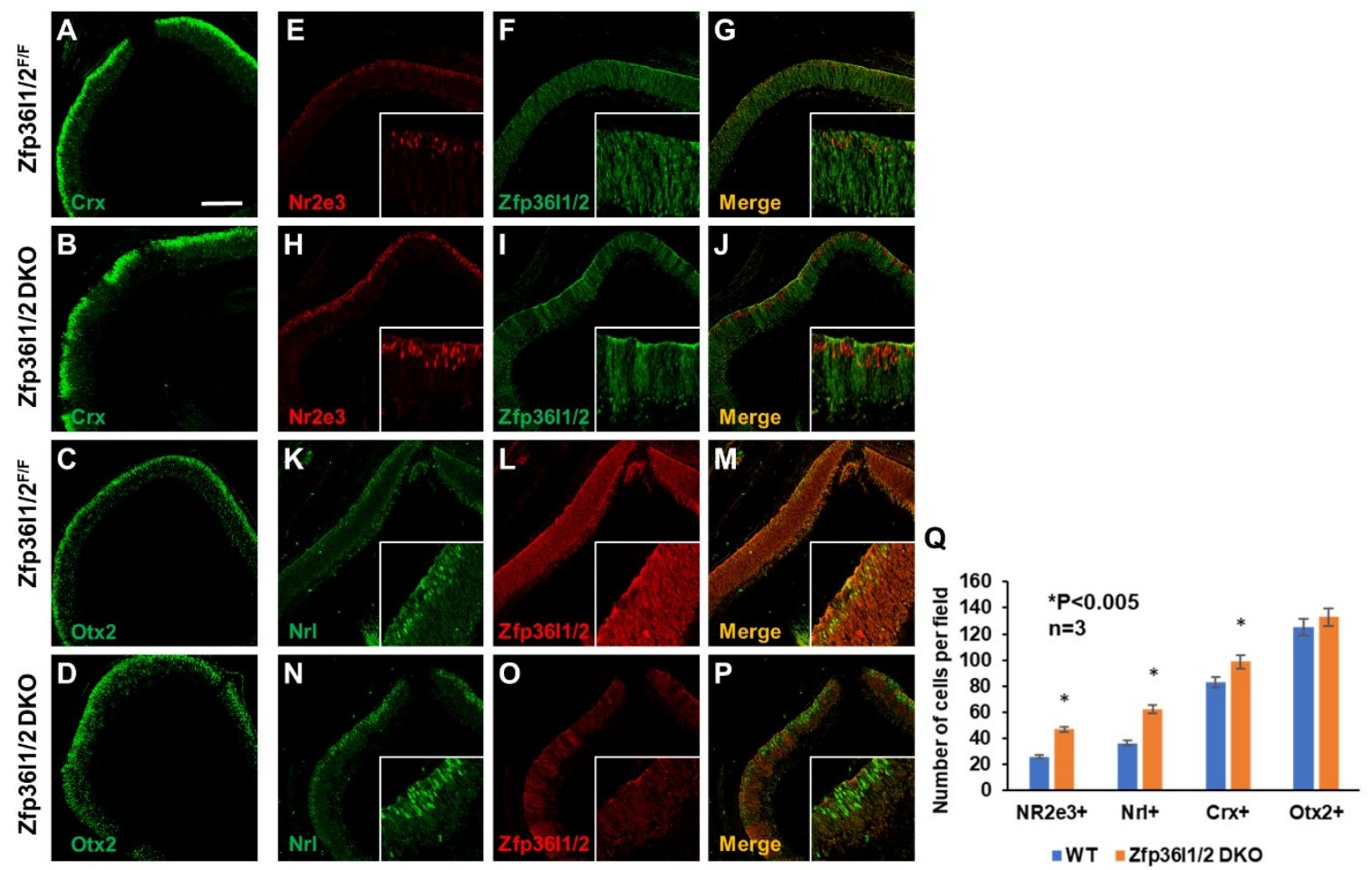

Figure 4. Rod differentiation increases in the Zfp36/1/2 DKO retina at E17.5. A-C. Immunostaining of $\mathrm{Crx}(\mathbf{A}, \mathbf{B})$ and $\mathrm{Otx2}$ (C, D), two photoreceptor markers, shows only moderate increases for these two markers (see $\mathbf{Q})$ in the DKO retina. E-J Co-immunostaining for Nr2e3 (red) and Zfp36/1/2 on control and Zfp36/1/2 DKO retinal sections. Note the increased $\mathrm{Nr2e} 3$ positive cells in regions where Zfp36/1 and Zfp36/2 are deleted (insets in H-J). K-P. Co-immunostaining of $\mathrm{Nrl}$ (green) and Zfp36l1/2 on E17.5 control and DKO retinal sections. Like Nr2e3, the increase in Nrl positive cells mostly occurs in areas where Zfp36/1 and Zfp36/1 are deleted (inlets in N-P). Q. Quantitation of positive cells for the four markers. Error bars indicate \pm SD. The scale bar in $\mathbf{A}$ is $150 \mu \mathrm{m}$ and applies to all panels. 

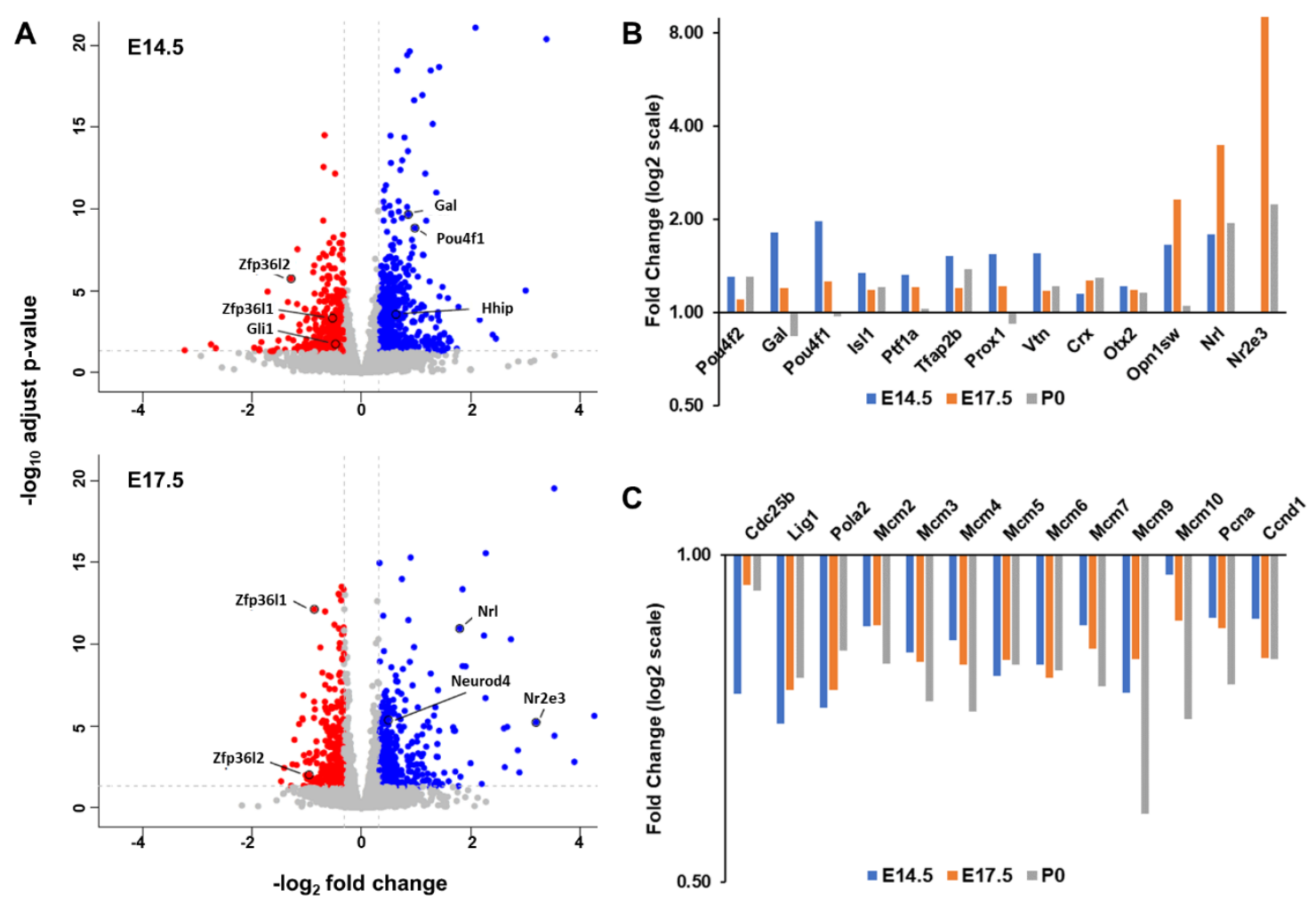

Figure 5. RNA-seq confirms increased differentiation of multiple cell types and reduced RPC proliferation. A. Volcano plots depicting down- (red) and upregulated (blue) genes in the Zfp36/1/2 DKO retina at E14.5 and 17.5. As expected, Zfp36/1 and Zfp36/2 were among the downregulated genes at both stages. A few marker genes for RGCs ( $\mathrm{Gal}$ and Pou4f1) and photoreceptors ( $\mathrm{Nrl}$, Neurod4, and Nr2e3) are highlighted at E14.5 and E17.5 respectively. Two genes of the Shh pathway, Hhip and Gli1, are also highlighted on the E14.5 plot. B. Upregulation of example marker genes for different cell types in the DKO retina at the three developmental stages. The y axis depicts fold change (mutant/wild-type) in log2 scale. C. Downregulation of multiple genes involved in cell cycle progression. Note that in both B and C, except for Otx2, all displayed genes have $a \geq 1.25$ fold change and an adjusted $p$ value of $\leq 0.05$ at least one of the three time points (see Suppl. Tables 1-3 for details). 
A

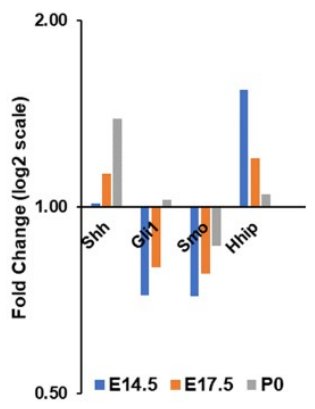

B

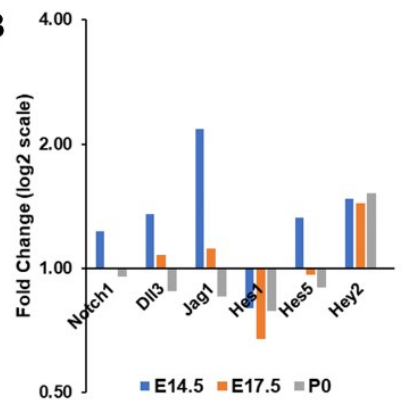

E

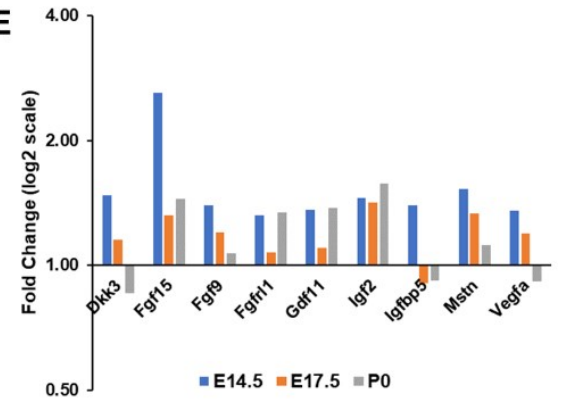

C

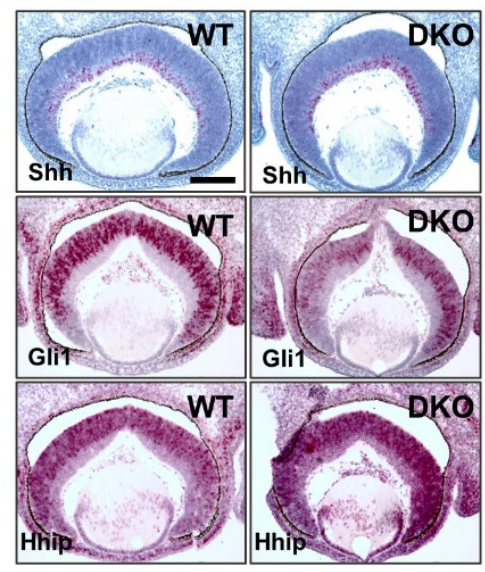

D

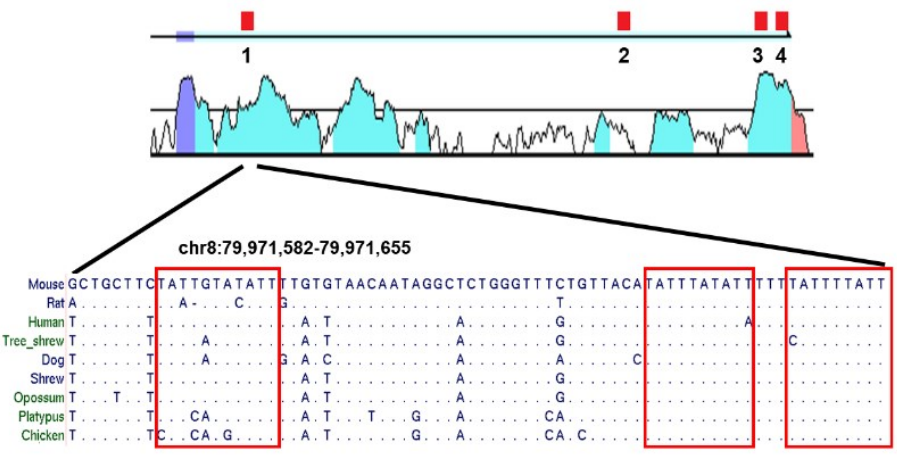

Figure 6. Multiple signaling pathways are influenced by Zfp36I1 and Zfp36I2. A. Multiple genes of the Shh pathway are affected in the Zfp36/1/2 DKO retina at E14.5. The downregulation of Gli1 and Smo indicates that the pathway is downregulated. This may be caused by the upregulation of Hhip, which encodes an Shh antagonist. B. Changes of genes of the Notch pathway. Noticeable changes in this pathway include upregulation of Notch1 and two of its ligand genes, DII3 and Jag1, downregulation of Hes1, and upregulation of Hes5. C. Confirmation of changes of the Shh pathway genes by in situ hybridization. Note that Shh is expressed in RGCs, whereas Gli1 and Hhip are expressed in RPCs. Red staining is in situ hybridization signal, and blue is hematoxylin counterstaining. The scale bar is $150 \mu \mathrm{m}$. D. Four regions containing conserved AU-rich elements (AREs) are present in the 3' UTR of Hhip mRNA. Top shows the structure of the last exon and positions of the four regions: purple is the coding region of the last exon, and light blue is 3' UTR. Positions of AREs are marked as red boxes and numbered. The middle track displays the conserved regions between mouse and human Hhip 3' UTRs from the Vista Genome Browser (pipeline.Ibl.gov). All four ARE containing regions are highly conserved between human and mouse. Bottom shows sequence alignments of region 1 from nine vertebrate species. Identical bases in species other than the mouse are as dots. This region contains three ARE motifs as indicated by red boxes, all highly conserved. E. Upregulation of a set of genes encoding secreted proteins in the DKO retina. 

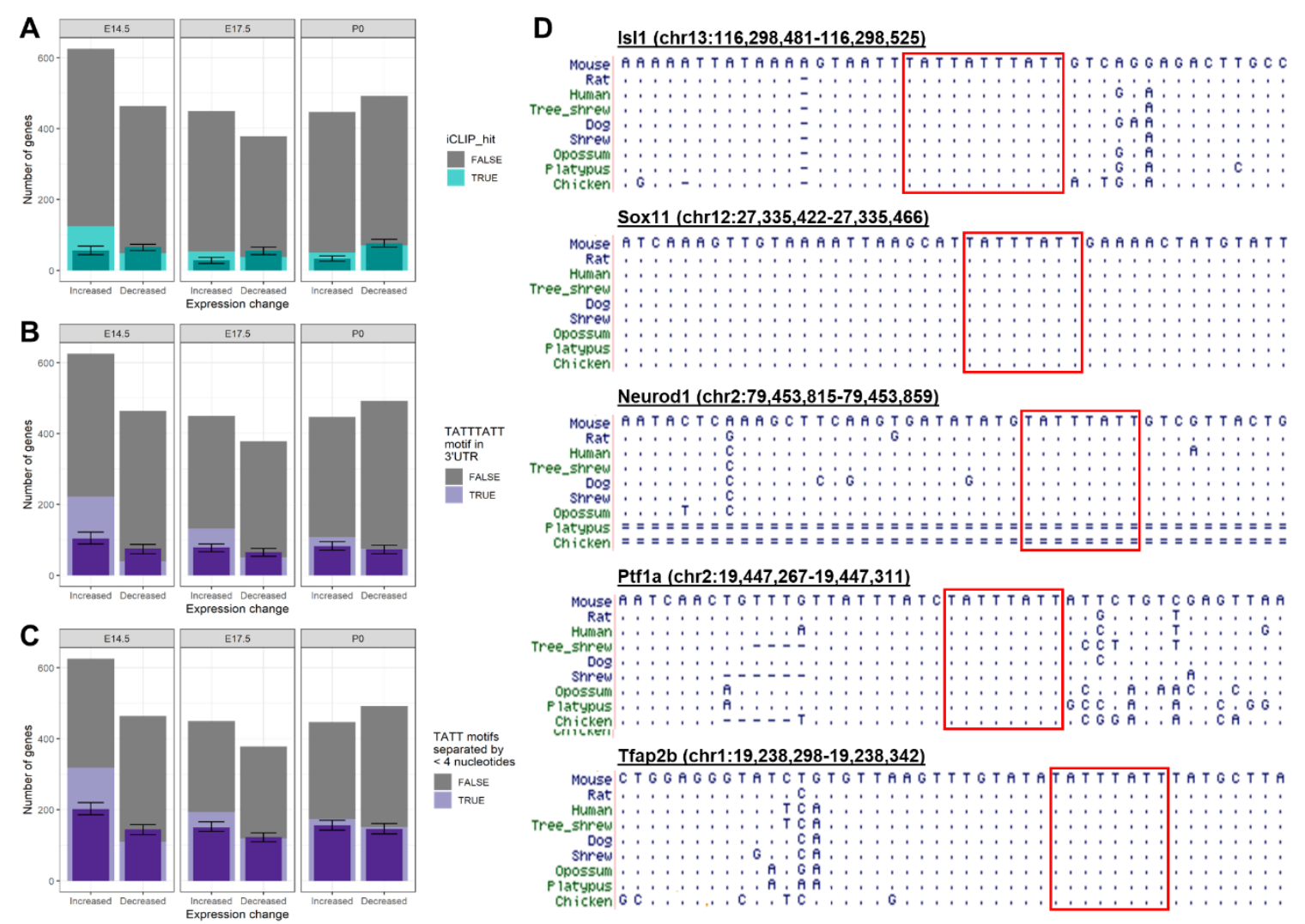

Figure 7. Upregulated genes are enriched with potential Zfp36I1 and Zfp36I2 targets. A. Enrichment analysis of the thymus target genes in the six differentially expressed gene lists (up- and downregulated genes for each stage). The y axis is gene numbers. The grey bars are the total numbers of DEGs on each list. Light turquoise bars show the number of genes that are the thymus targets in each DEG list. Dark turquoise and error bars show the median and 5th/95th percentile for the number of targets in the 100 control gene sets. B. C. Enrichment analysis of genes containing the TATTTATT motif $(B)$ and closely spaced ( $<4$ bases) TATT motifs $(\mathbf{C})$ in the six DEG gene lists. The grey bars are the total numbers of DEGs on each list. Light purple bars show the number of genes containing the motifs in each DEG list. Dark purple and error bars show the median and 5th/95th percentile for the number of genes containing the motifs in the corresponding control gene sets. D. Conservation of ARE motifs in genes encoding transcription factors that regulate retinal cell differentiation. Sequences of the 3' UTR regions of five transcription factor genes containing the ARE motifs are aligned for nine vertebrate species. Identical bases were in species other than the mouse are shown as dots. Dashes indicate gaps and double lines indicate unalignable sequences. Mouse genome coordinates ( $\mathrm{mm} 10$ ) are provided for sequences of each gene. Conserved ARE motifs are highlighted by red boxes. 

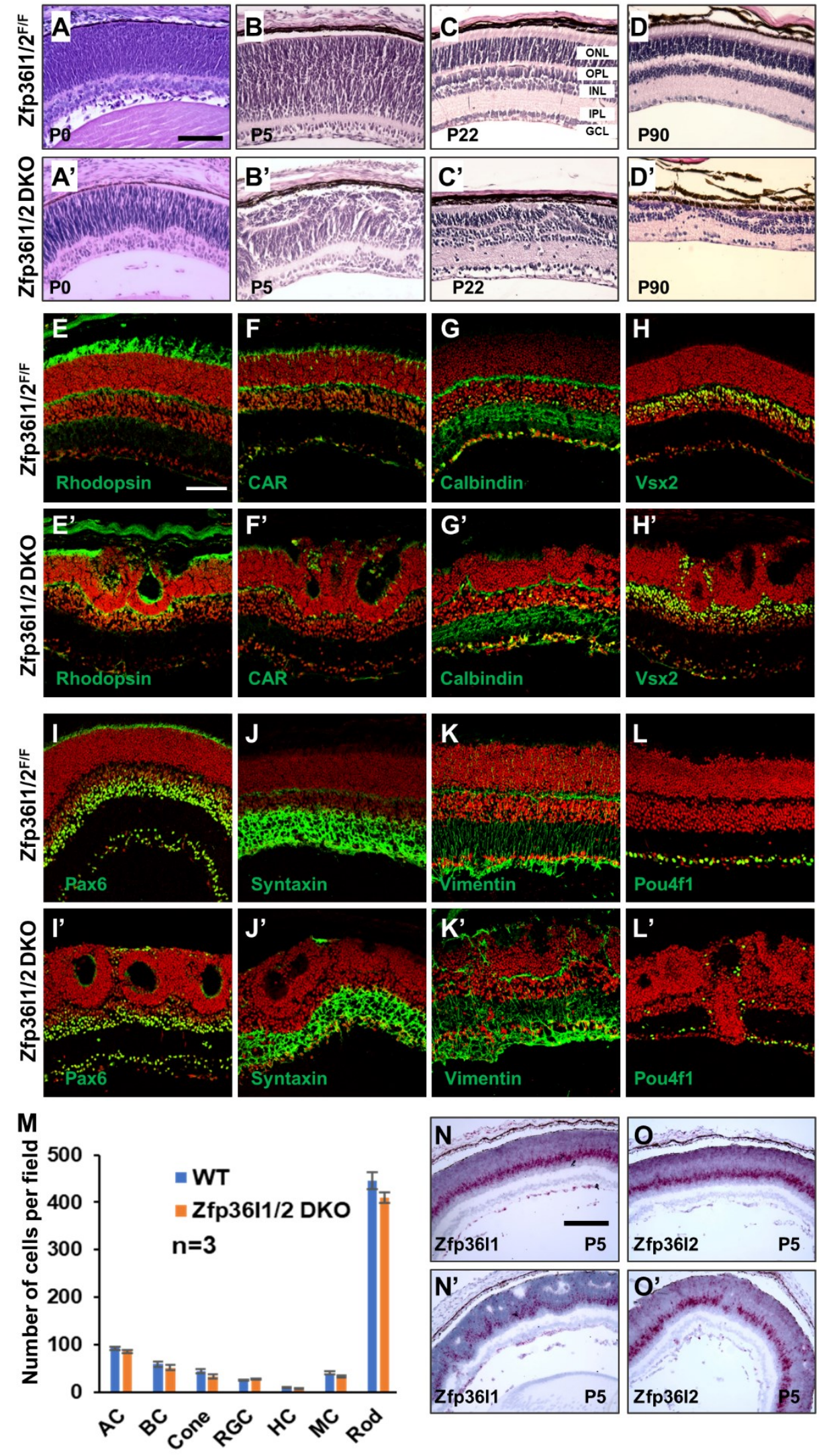
Figure 8. Postnatal Zfp36/1/2 DKO retina displays dysplasia and degeneration. A-D, A'-D'. H\&E staining of control (A-D) and Zfp36/1/2 DKO (A'D') retinal sections from P0, P5, P22, and P90 mice, respectively. E-L, E'-L'. Immunofluorescence labeling for cell type-specific markers to examine the formation of the seven retinal cell types in control (E-L) and Zfp36/1/2 DKO (E'-L') retinas (see text for details). Nuclei (red) were stained with propidium iodide. $\mathbf{M}$. Quantitation of positive cells in E-L and $\mathbf{E}^{\prime}$ - $\mathrm{L}^{\prime}$. Error bars indicate \pm SD. . N, O, N', O'. RNAscope in situ hybridization of P5 control (N, O) and DKO (N', O') P16 retinal sections for Zfp36/1 ( $\mathbf{N}$ and $\mathbf{N}^{\prime}$ ) and Zfp36/2 (O and $\mathbf{O}^{\prime}$ ). Note that despite the disruptions of the retinal structure in the DKO retina, only very small gaps of mutant cells can be seen. Scale bars: in A, $75 \mu \mathrm{m}$ (A-D'); in E, $150 \mu \mathrm{m}$ (E-L'); in $\mathbf{N}, 150 \mu \mathrm{m}$ (N-O'). 


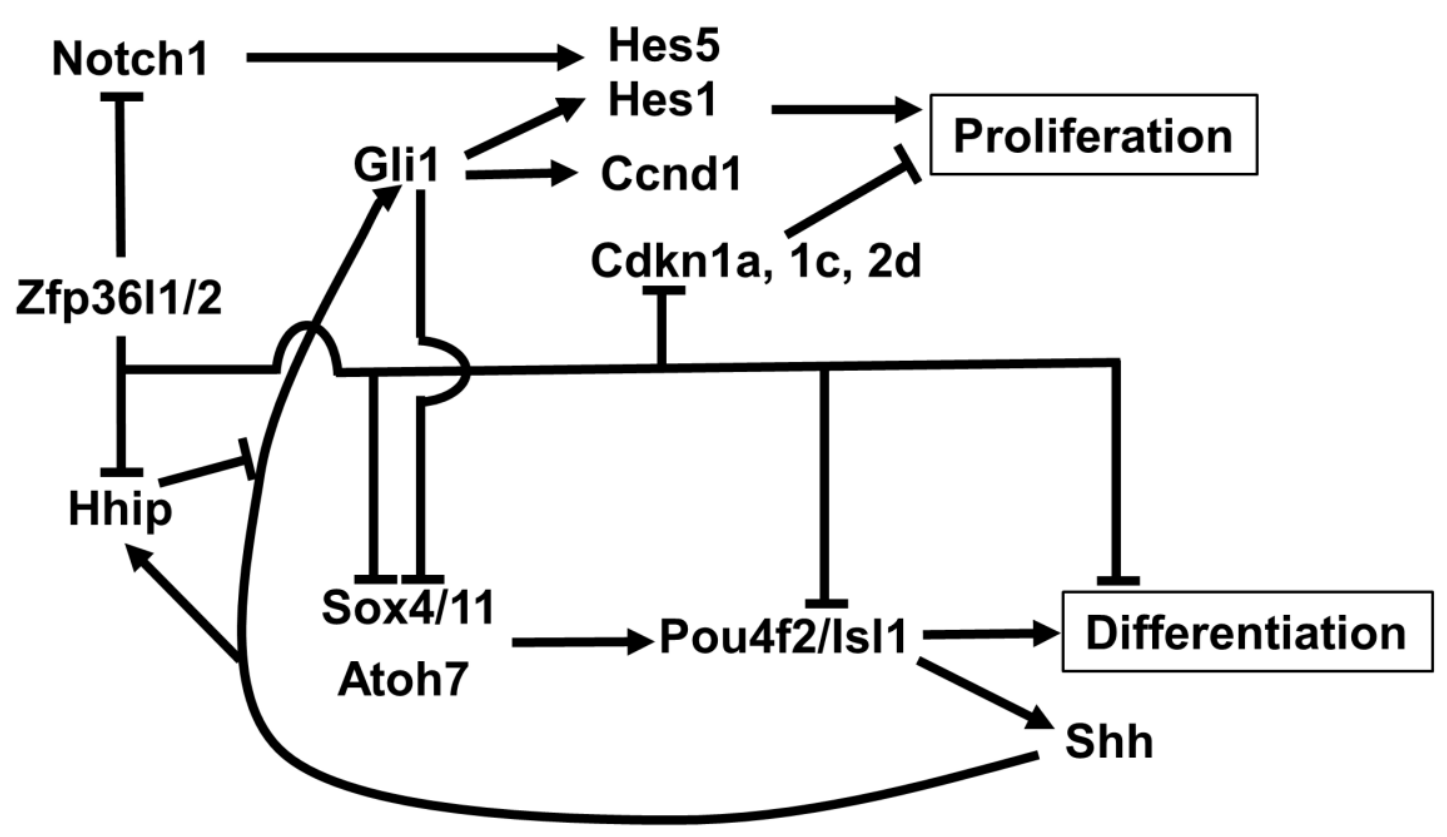

Figure 9. Zfp36I1 and Zfp36I2 represent an additional layer to the genetic network regulating the balance of proliferation and differentiation. In this network, proliferation and differentiation are regulated by distinct but interacting sets of regulators. For simplicity, only differentiation of retinal ganglion cells (RGCs) is shown. The Notch pathway is part of the regulatory mechanism promoting RPC proliferation and is turned off upon differentiation. Atoh7, Sox4/11, Pou4f2, and Isl1 are part of the regulatory mechanisms promoting RGC differentiation. While promoting differentiation, Atoh7, Sox4/11, Pou4f2, and Isl1 also activate Shh, and Shh feeds back on RPCs to promote proliferation and inhibit differentiation. Shh also activates its own antagonist Hhip, which is in turn inhibited by Zfp36l1 and Zfp36I2. Zfp36l1 and Zfp36l2 also inhibit Notch1 expression, but the outcome of this inhibition may be partially antagonized by the Shh pathway, as the Shh pathway activates Hes1, which is one of the downstream and effector genes of the Notch pathway. Further, Zfp36l1 and Zfp36l2 directly promote cell cycle progression by inhibiting cyclin dependent kinase inhibitors (Cdkn1a, Cdkn1c, Cdkn2d), and repress differentiation by negatively regulating the key transcription factors (e.g. Sox11, Isl1, and Pou4f2). 


\begin{tabular}{|c|c|c|c|c|}
\hline Term & Count & $\%$ & $\begin{array}{c}\text { Fold } \\
\text { Enrichment }\end{array}$ & FDR \\
\hline \multicolumn{5}{|l|}{ Upregulated genes } \\
\hline visual perception & 36 & 3.03 & 4.78 & 0.00 \\
\hline nervous system development & 61 & 5.13 & 2.85 & 0.00 \\
\hline neuron projection development & 31 & 2.61 & 3.93 & 0.00 \\
\hline neurotransmitter transport & 17 & 1.43 & 6.66 & 0.00 \\
\hline cell adhesion & 63 & 5.30 & 2.29 & 0.00 \\
\hline retina development in camera-type eye & 22 & 1.85 & 4.62 & 0.00 \\
\hline ion transport & 66 & 5.56 & 1.99 & 0.00 \\
\hline regulation of membrane potential & 21 & 1.77 & 3.82 & 0.00 \\
\hline regulation of dopamine secretion & 8 & 0.67 & 9.41 & 0.01 \\
\hline axonogenesis & 20 & 1.68 & 3.30 & 0.01 \\
\hline photoreceptor cell maintenance & 12 & 1.01 & 5.29 & 0.02 \\
\hline axon guidance & 24 & 2.02 & 2.84 & 0.02 \\
\hline $\begin{array}{l}\text { chemical synaptic transmission } \\
\text { regulation of long-term neuronal synaptic }\end{array}$ & 26 & 2.19 & 2.67 & 0.02 \\
\hline plasticity & 10 & 0.84 & 6.30 & 0.03 \\
\hline \multicolumn{5}{|l|}{ Downregulated genes } \\
\hline translation & 75 & 7.30 & 3.67 & 0.00 \\
\hline $\begin{array}{l}\text { DNA replication } \\
\text { cellular response to DNA damage }\end{array}$ & 33 & 3.21 & 5.26 & 0.00 \\
\hline stimulus & 61 & 5.94 & 2.85 & 0.00 \\
\hline DNA repair & 50 & 4.87 & 3.08 & 0.00 \\
\hline cytoplasmic translation & 16 & 1.56 & 9.23 & 0.00 \\
\hline nucleosome assembly & 26 & 2.53 & 4.90 & 0.00 \\
\hline DNA replication initiation & 11 & 1.07 & 8.99 & 0.00 \\
\hline ribosomal small subunit biogenesis & 10 & 0.97 & 10.32 & 0.00 \\
\hline cell cycle & 63 & 6.13 & 2.01 & 0.00 \\
\hline rRNA processing & 23 & 2.24 & 3.58 & 0.00 \\
\hline ribosomal small subunit assembly & 10 & 0.97 & 9.34 & 0.00 \\
\hline metabolic process & 50 & 4.87 & 2.12 & 0.00 \\
\hline ribosomal large subunit biogenesis & 9 & 0.88 & 7.35 & 0.03 \\
\hline
\end{tabular}

